Supplement of The Cryosphere, 13, 2537-2556, 2019

https://doi.org/10.5194/tc-13-2537-2019-supplement

(C) Author(s) 2019. This work is distributed under

the Creative Commons Attribution 4.0 License.

(c) (1)

Supplement of

\title{
Changes of the tropical glaciers throughout Peru between 2000 and 2016 - mass balance and area fluctuations
}

Thorsten Seehaus et al.

Correspondence to: Thorsten Seehaus (thorsten.seehaus@fau.de)

The copyright of individual parts of the supplement might differ from the CC BY 4.0 License. 

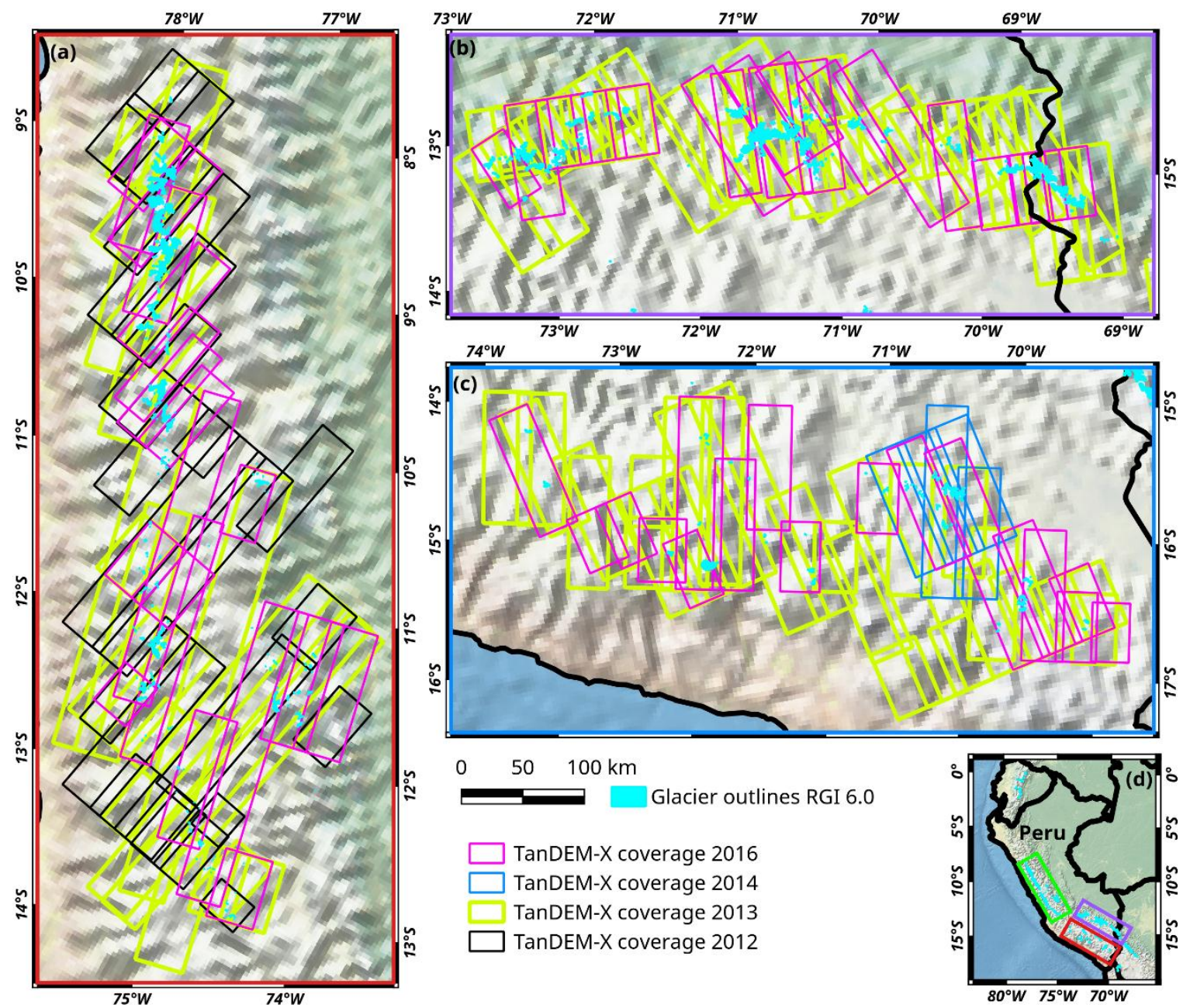

Figure S1. Coverage of the studied areas by TanDEM-X data. Panels (a-c): glacier subregions in Peru according to Sagredo and Lowell (2012); (a) subregion R1: northern wet outer tropics; (b) subregion R2: southern wet outer tropics; (c) subregion R3: dry outer tropics. Panel (d): overview map of Peru. Coloured rectangles indicate the locations of the subregions (same frame colours). Light blue areas: glacier coverage based on RGI 6.0. (c) Natural Earth 


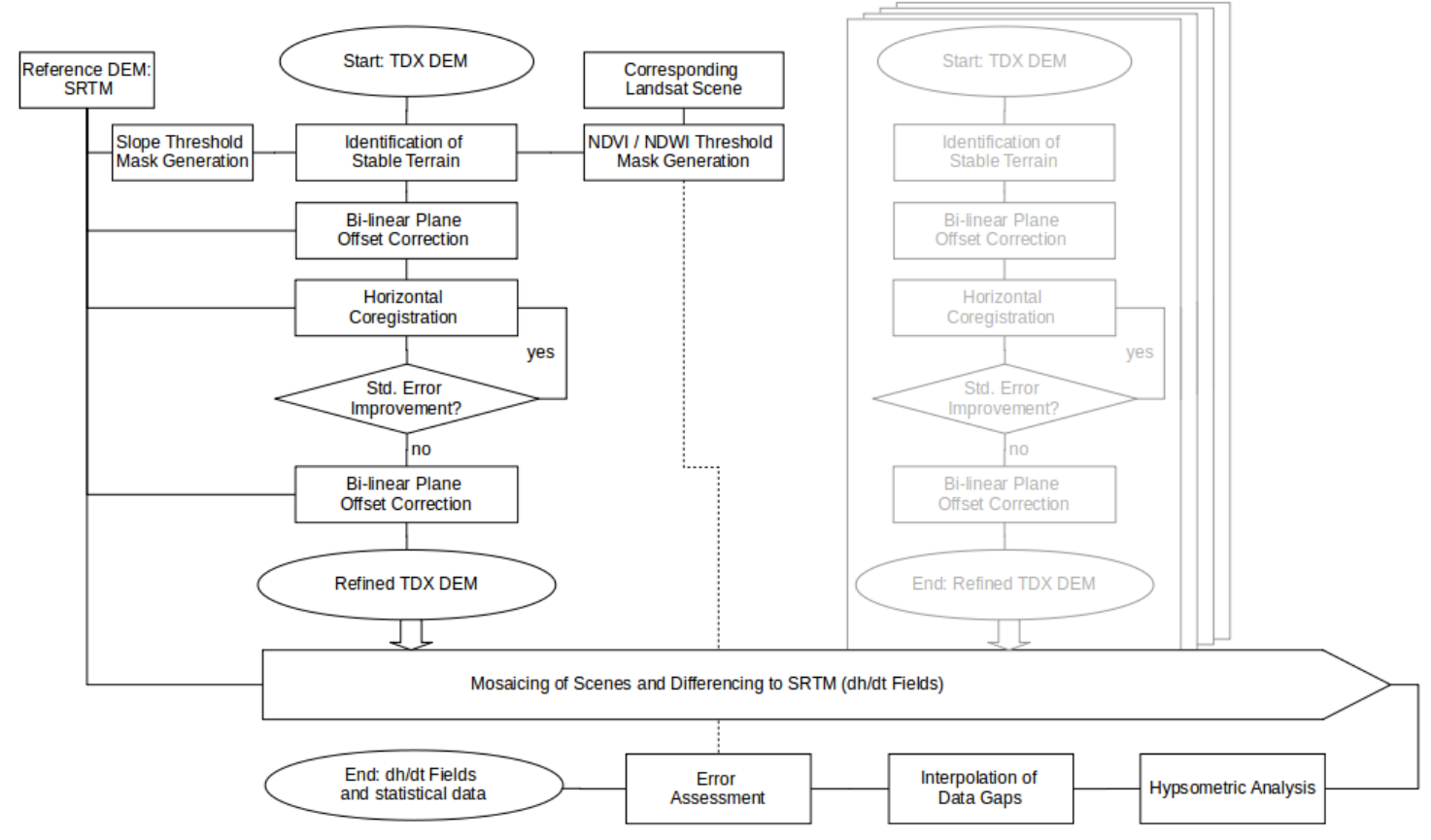

Figure S2. Flow chart of processing chain to perform coregistration, mosaicking, gap filling and error evaluation of TanDEM-X DEMs 


\section{R2-2000-2016}

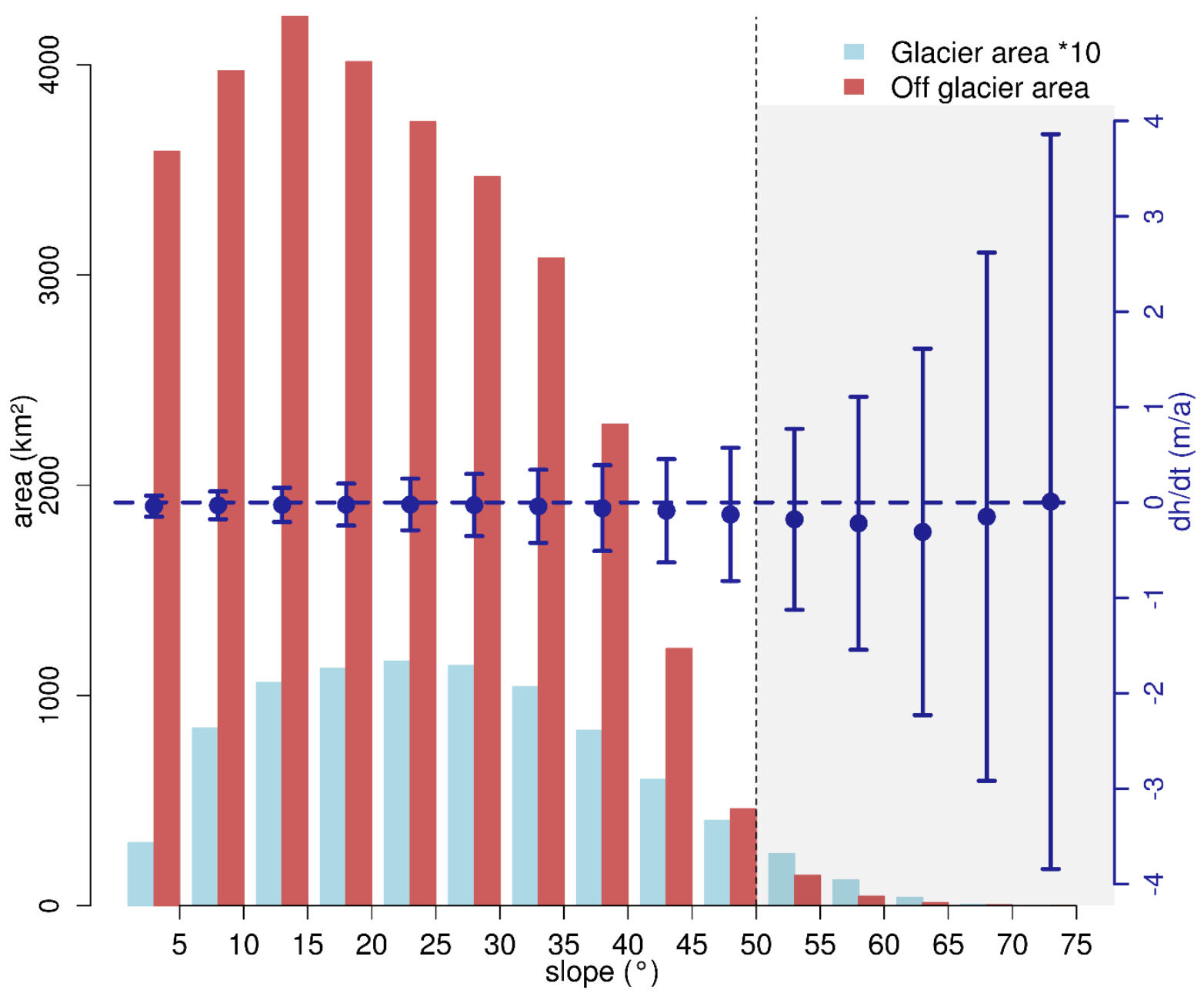

Figure S3. Off-(red) and on-glacier (light blue) area and off-glacier elevation change (blue dots) distributions in dependency on slope in subregion R2 for the period 2000-2016. Error bars represent NMAD of $\Delta \mathrm{h} / \Delta \mathrm{t}$ values in the individual slope interval. Dotted line indicates the applied slope threshold (see Section 4.2). Glacier area measurements are based on the glacier outlines from 2000. Note: For better representation, on-glacier areas are scaled by a factor of 10 . 
R3-2000-2016

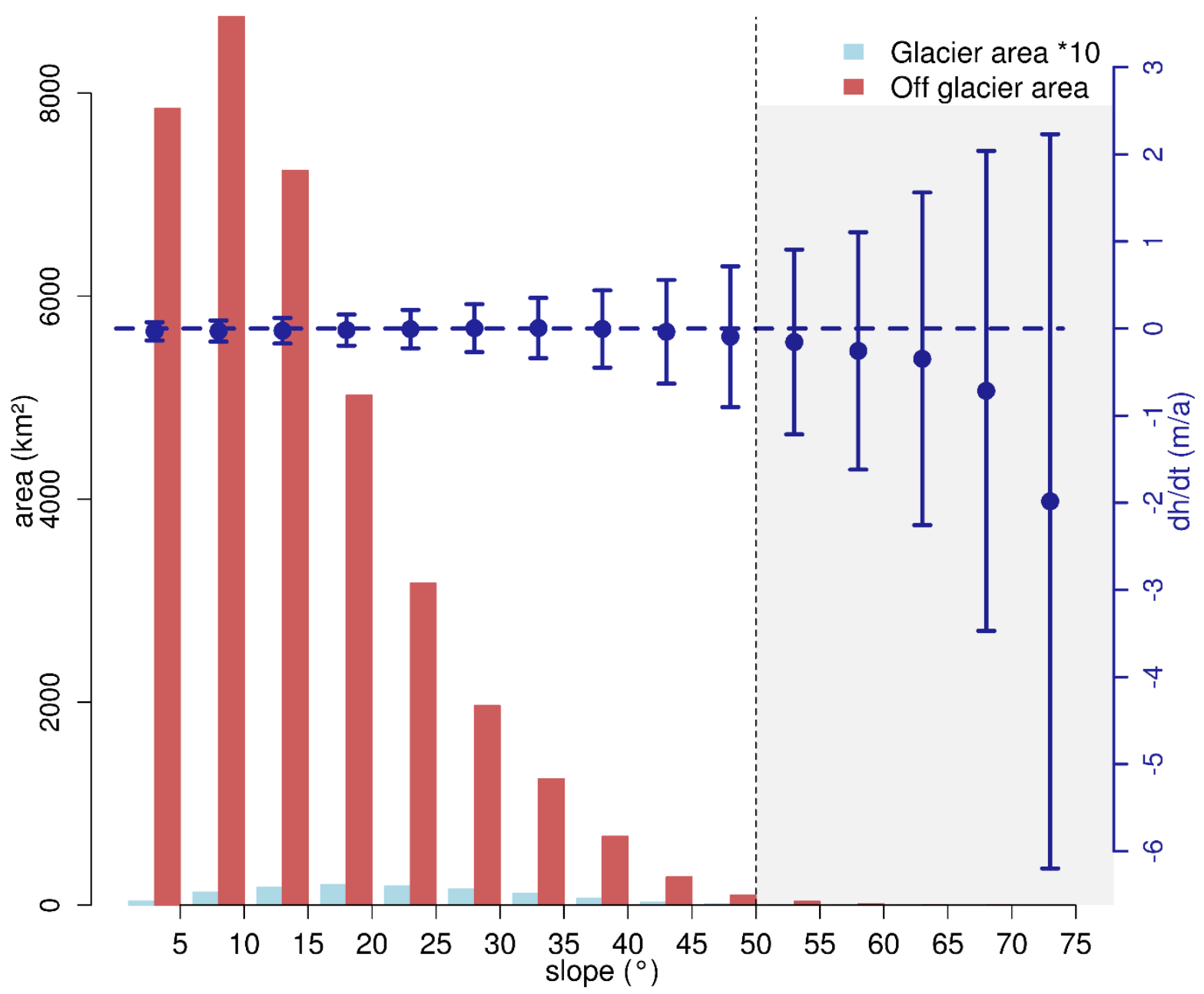

Figure S4. Off-(red) and on-glacier (light blue) area and off-glacier elevation change (blue dots) distributions in dependency on slope in subregion R3 for the period 2000-2016. Error bars represent NMAD of $\Delta \mathrm{h} / \Delta \mathrm{t}$ values in the individual slope interval. Dotted line indicates the applied slope threshold (see Section 4.2). Glacier area measurements are based on the glacier outlines from 2000. Note: For better representation, on-glacier areas are scaled by a factor of 10 . 

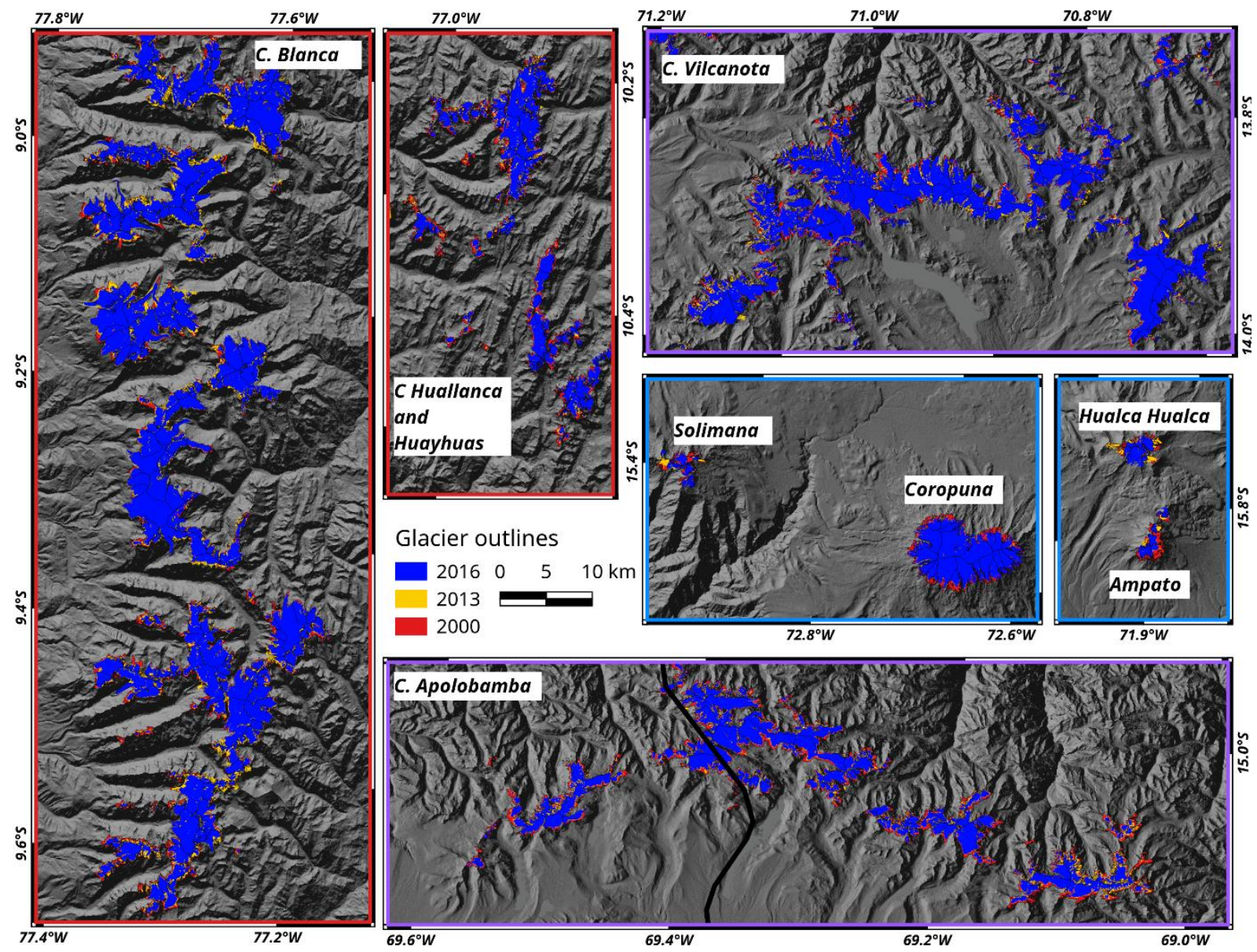

Figure S5. Glacier outlines at example mountain ranges in all three subregions in the years 2000, 2013 and 2016. The frame colour of the panels indicates the subregions (see Figure 1, main manuscript). Background: SRTM DEM hillshade ( NASA 


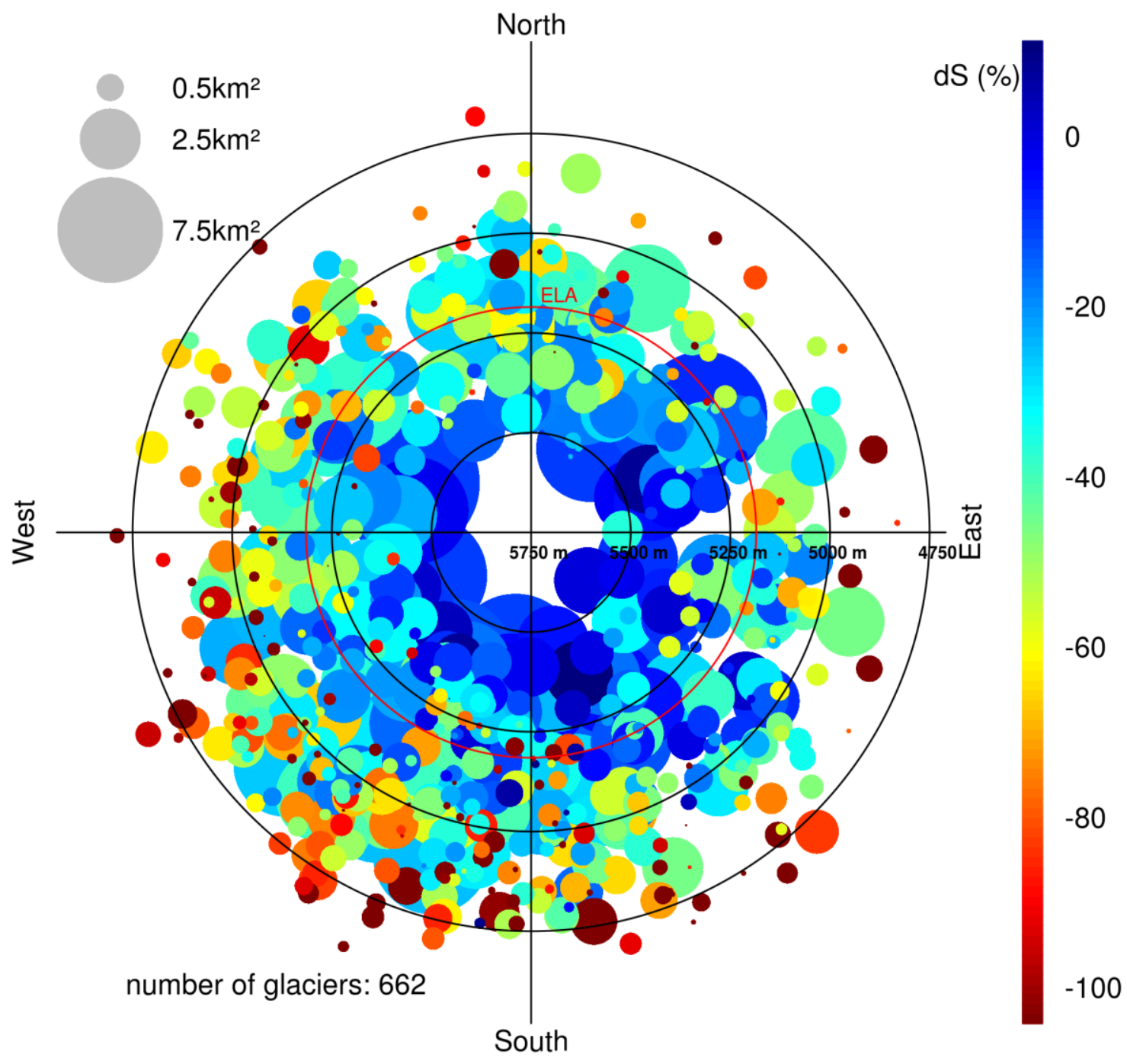

Figure S6. Polar plot of relative area changes (dot colour) in subregion R2 in the period 2000-2016 of individual glaciers. Dot size: glacier size in 2000; Radius: median elevation; Orientation: mean aspect. Red circle: equilibrium line altitude (ELA), see also Table S3. 


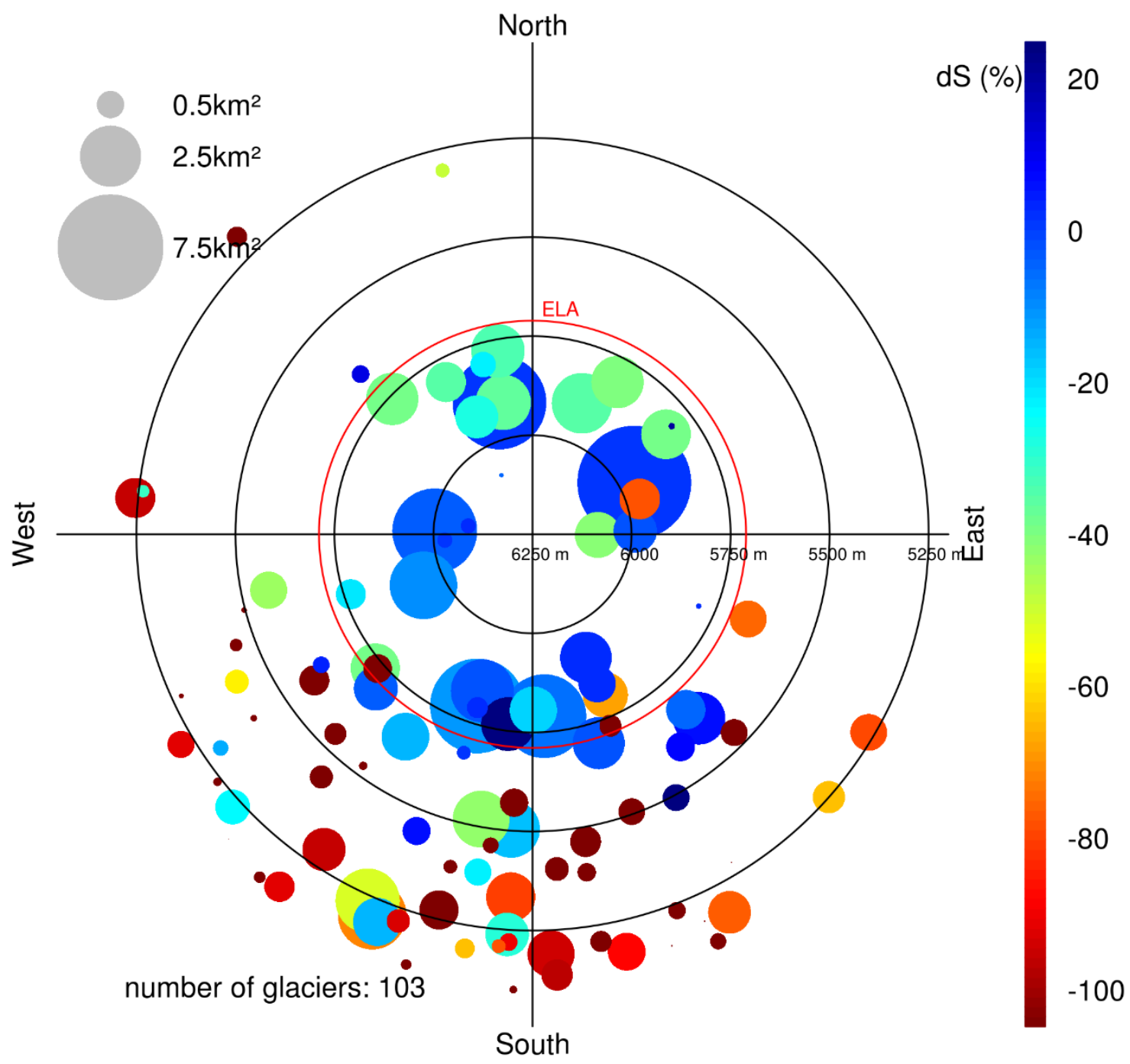

Figure S7. Polar plot of relative area changes (dot colour) in subregion R3 in the period 2000-2016 of individual glaciers. Dot size: glacier size in 2000; Radius: median elevation; Orientation: mean aspect. Red circle: equilibrium line altitude (ELA), see also Table S3. 


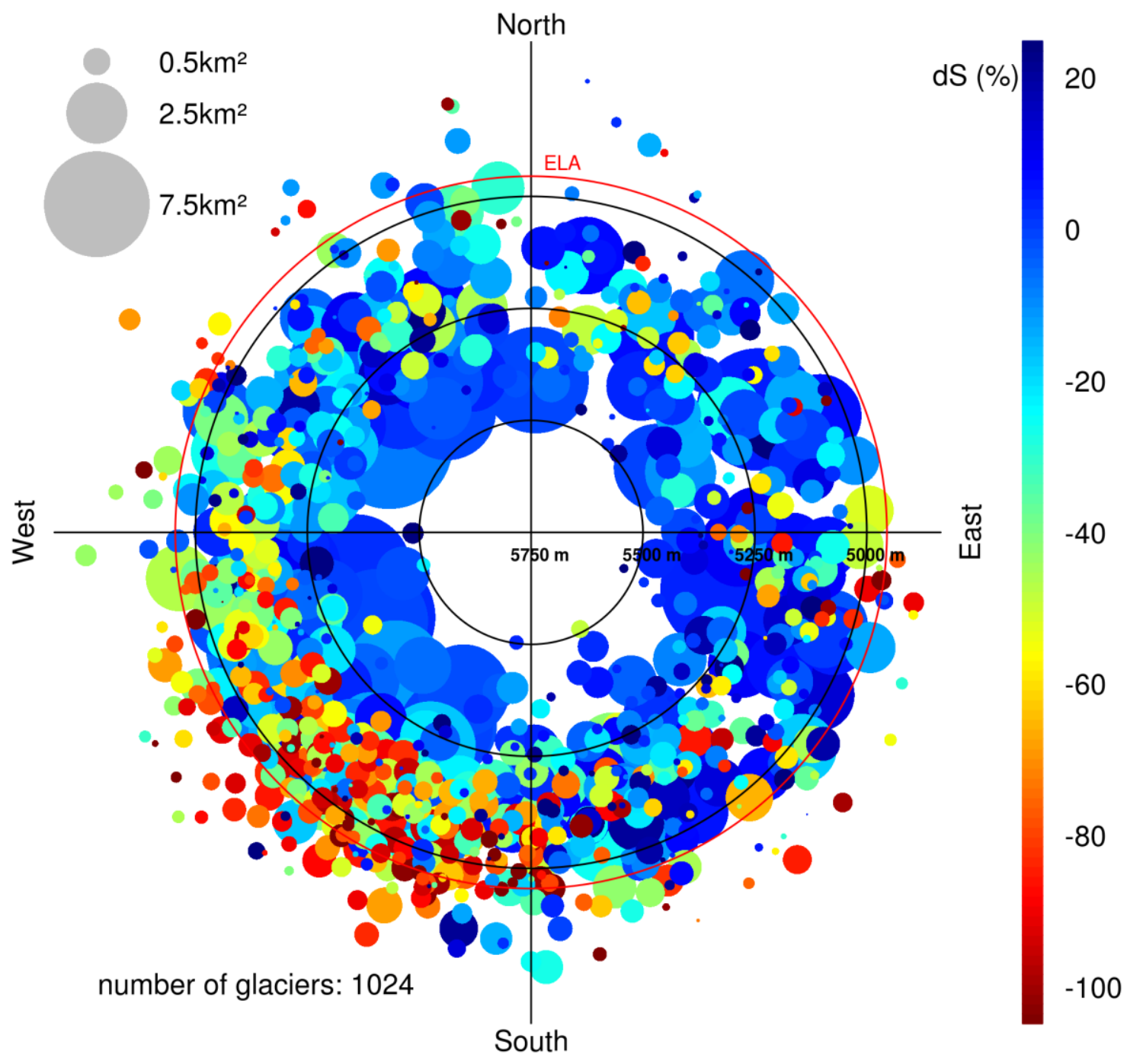

Figure S8. Polar plot of relative area changes (dot colour) in subregion R1 in the period 2000-2013 of individual glaciers. Dot size: glacier size in 2000; Radius: median elevation; Orientation: mean aspect. Red circle: equilibrium line altitude (ELA), see also Table S3. 


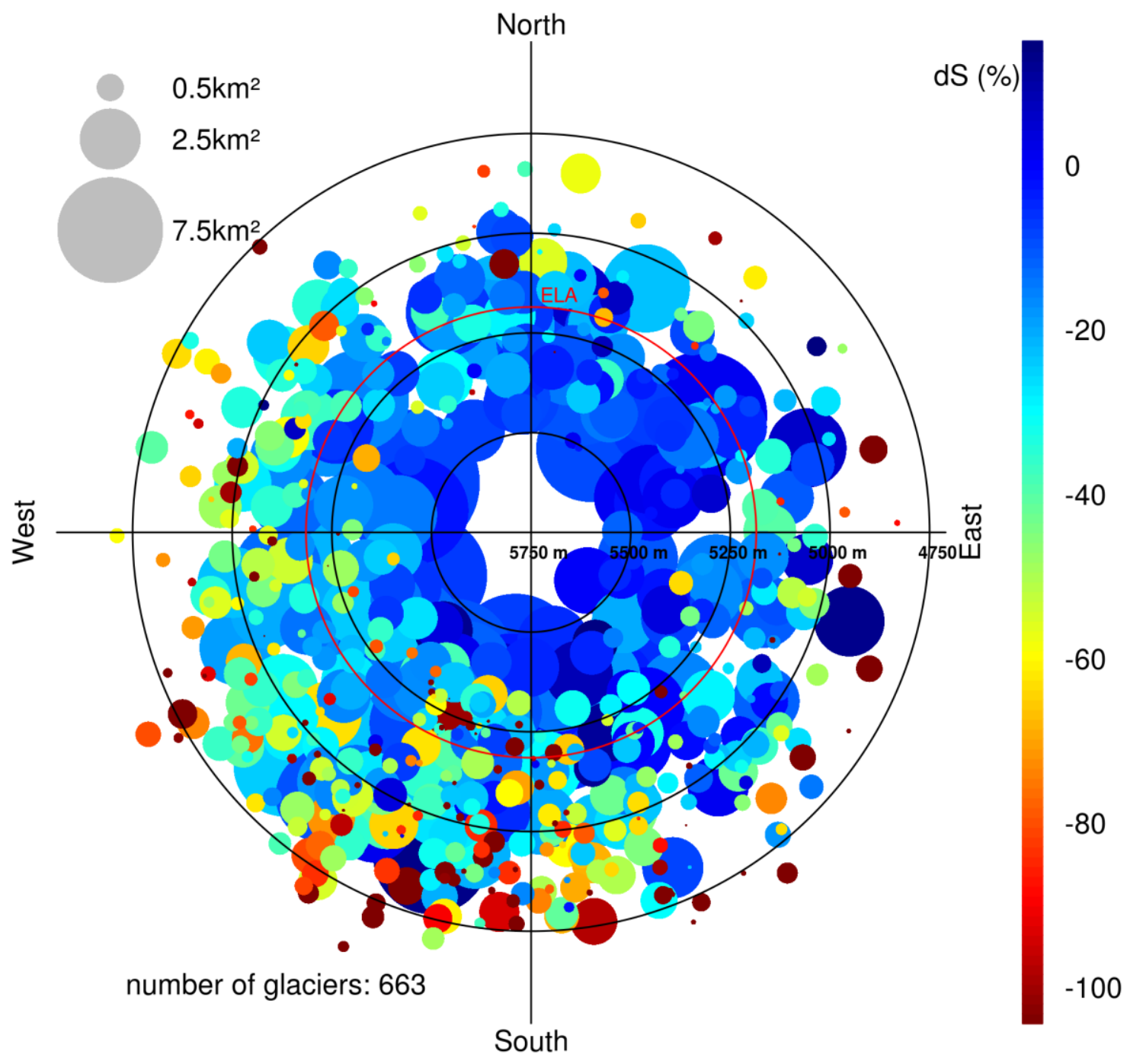

Figure S9. Polar plot of relative area changes (dot colour) in subregion R2 in the period 2000-2013 of individual glaciers. Dot size: glacier size in 2000; Radius: median elevation; Orientation: mean aspect. Red circle: equilibrium line altitude (ELA), see also Table S3. 


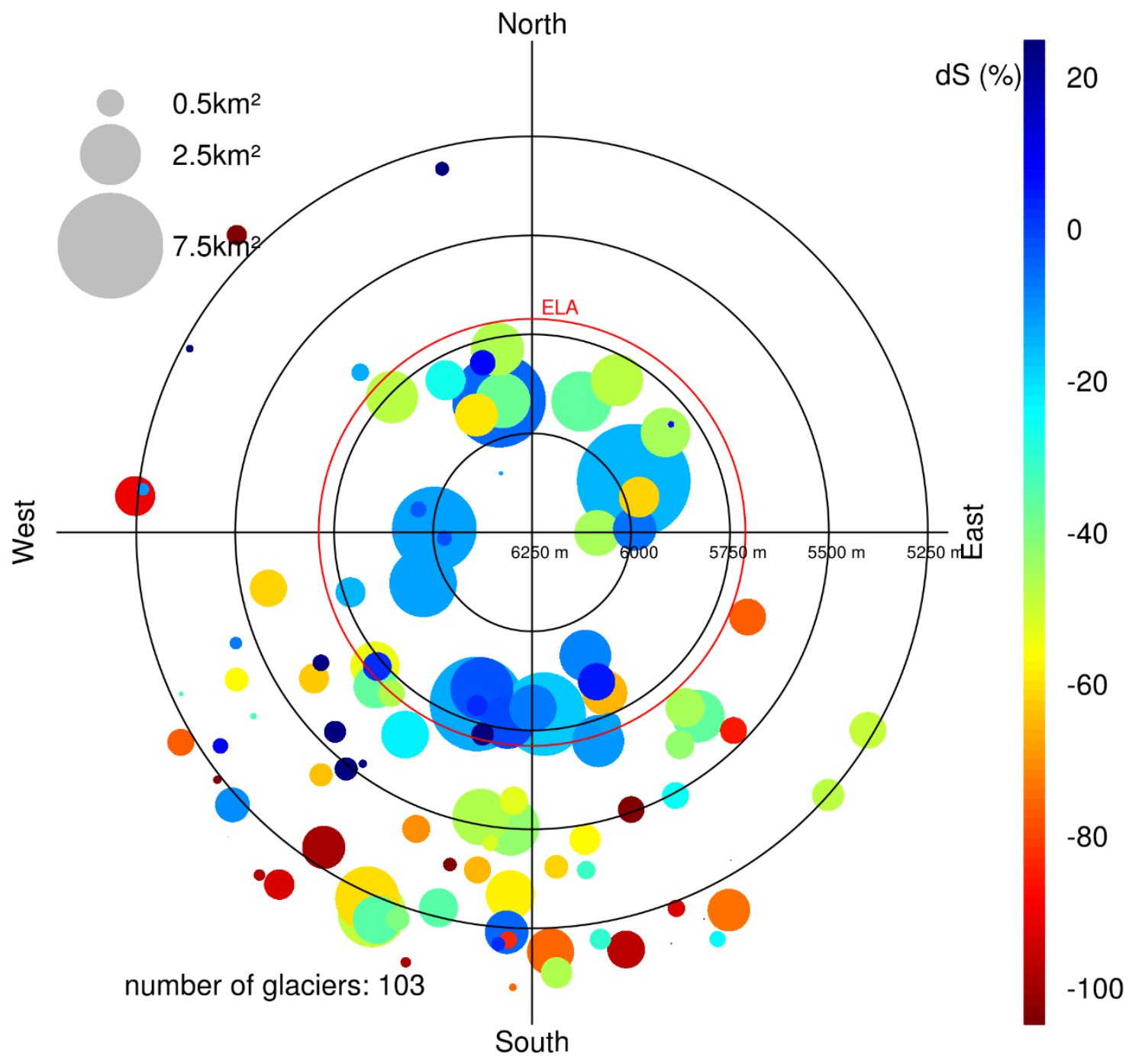

Figure S10. Polar plot of relative area changes (dot colour) in subregion R3 in the period 2000-2013 of individual glaciers. Dot size: glacier size in 2000; Radius: median elevation; Orientation: mean aspect. Red circle: equilibrium line altitude (ELA), see also Table S3. 


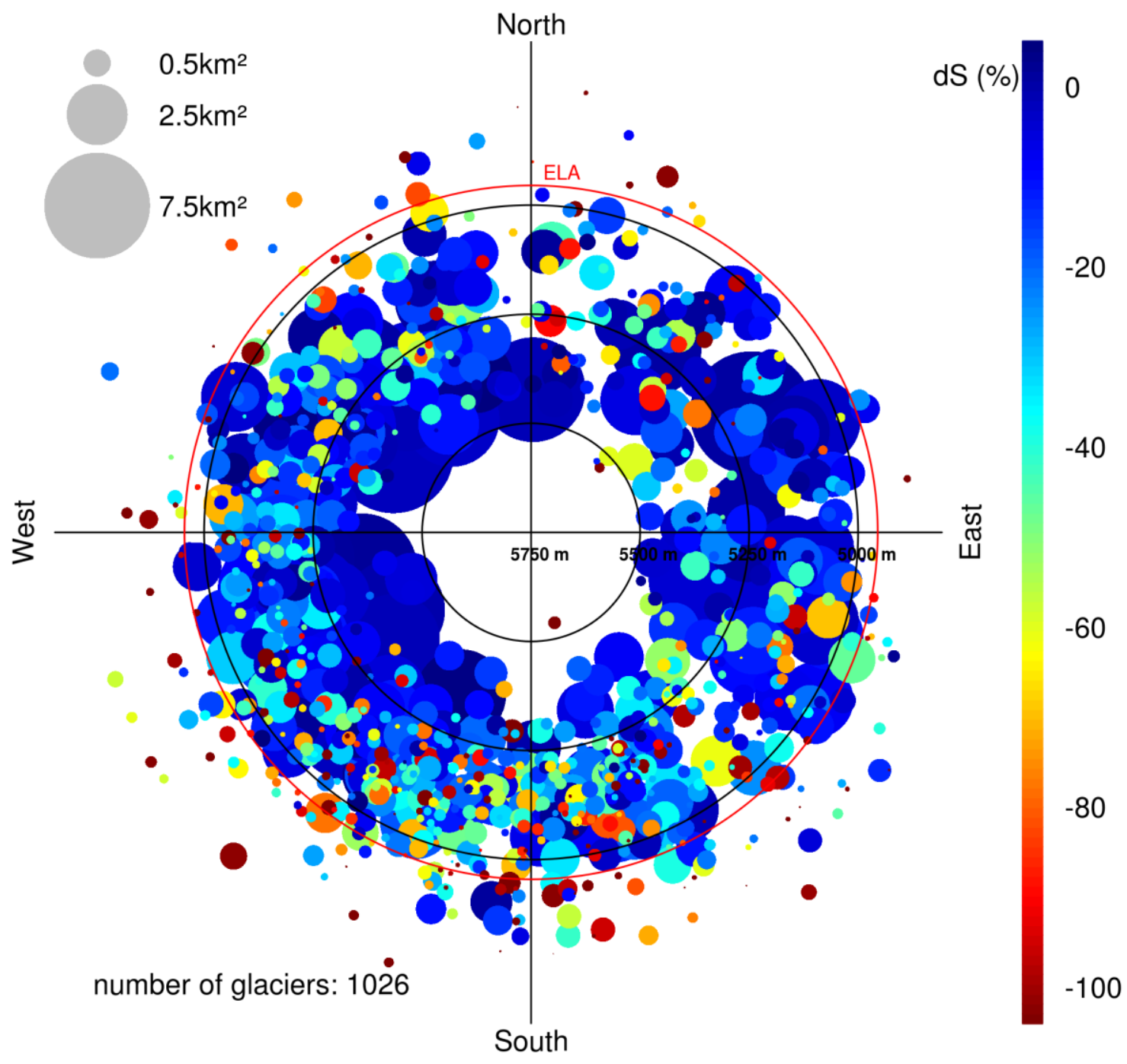

Figure S11. Polar plot of relative area changes (dot colour) in subregion R1 in the period 2013-2016 of individual glaciers. Dot size: glacier size in 2013; Radius: median elevation; Orientation: mean aspect. Red circle: equilibrium line altitude (ELA), see also Table S3. 


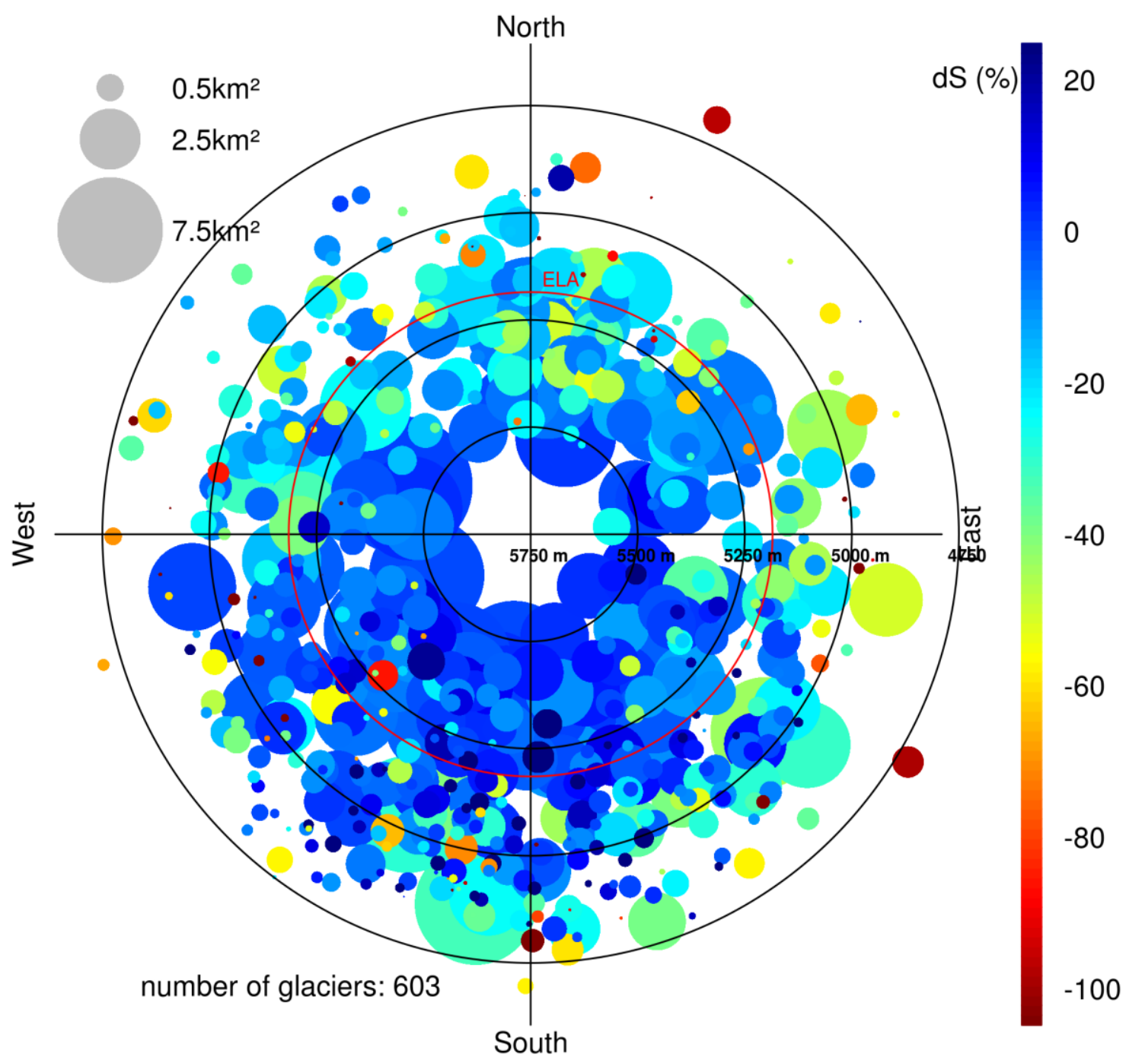

Figure S12. Polar plot of relative area changes (dot colour) in subregion R2 in the period 2013-2016 of individual glaciers. Dot size: glacier size in 2013; Radius: median elevation; Orientation: mean aspect. Red circle: equilibrium line altitude (ELA), see also Table S3. 


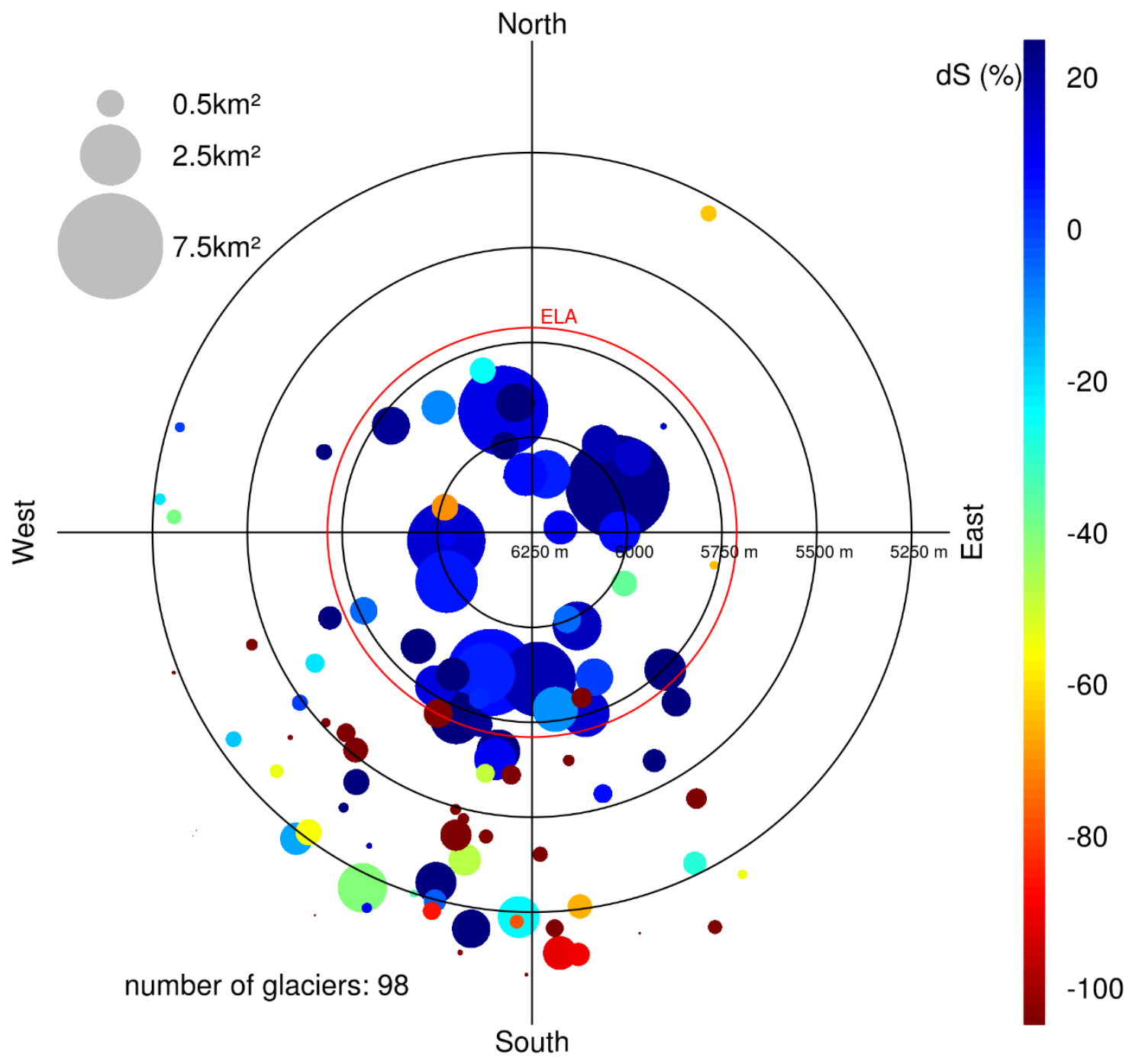

Figure S13. Polar plot of relative area changes (dot colour) in subregion R3 in the period 2013-2016 of individual glaciers. Dot size: glacier size in 2013; Radius: median elevation; Orientation: mean aspect. Red circle: equilibrium line altitude (ELA), see also Table S3. 


\section{R2-2000-2016}

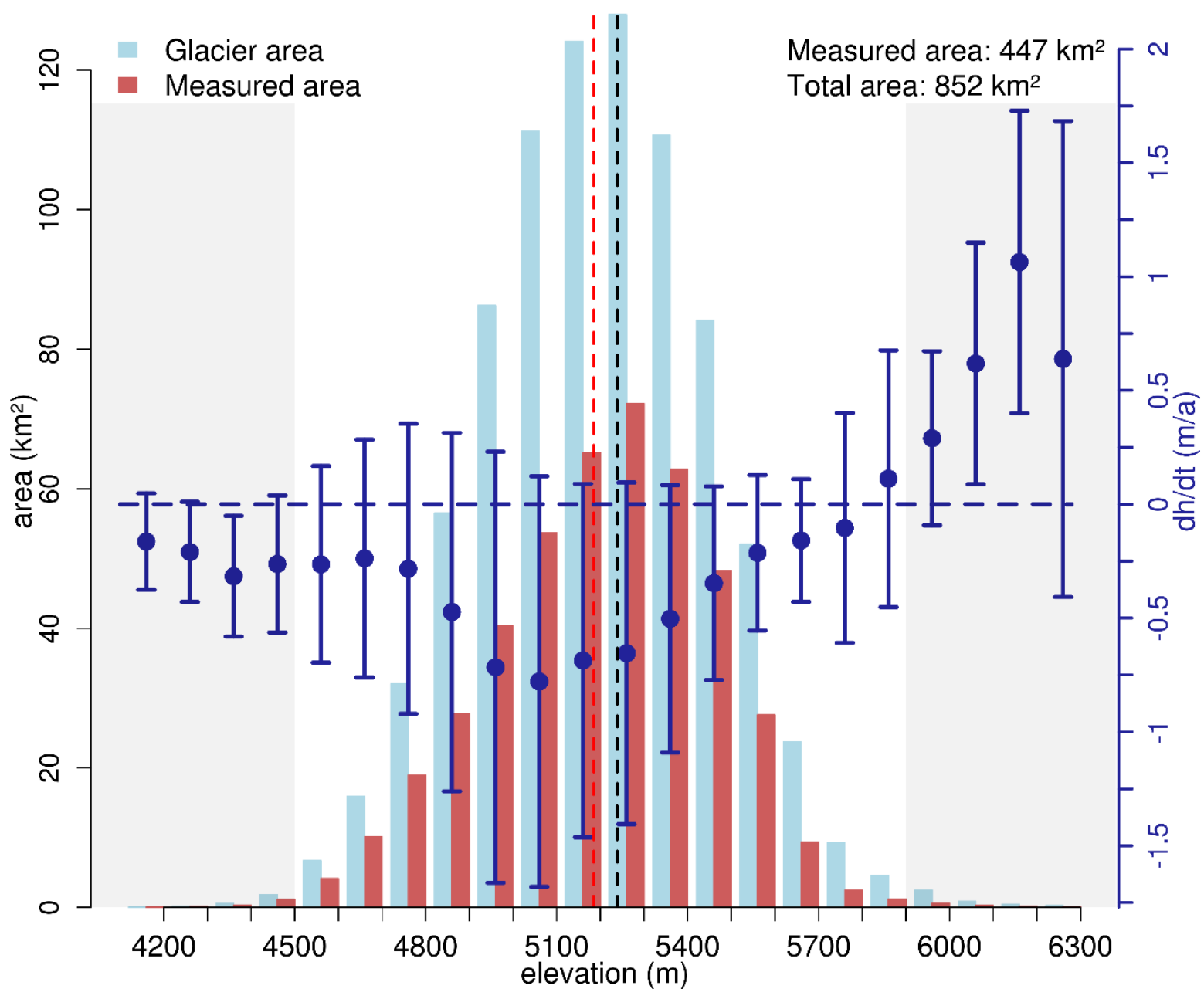

Figure S14. Hypsometric distribution of measured (red bars) and total (light blue bars) glacier area with elevation in subregion R2 in the interval 2000-2016. Blue dots represent the mean $\Delta \mathrm{h} / \Delta \mathrm{t}$ value in each elevation interval. Error bars indicate NMAD of $\Delta \mathrm{h} / \Delta \mathrm{t}$ for each hypsometric bin. Grey areas mark the lower and upper 1\% quantile of the glacier area distribution. Black dashed line: mean glacier elevation; Red dashed line: equilibrium line altitude (ELA), see also Table S3. Area measurements are based on the glacier outlines from 2000, considering only regions with slopes below applied slope threshold (50, see Section 4.2) 


\section{R3-2000-2016}

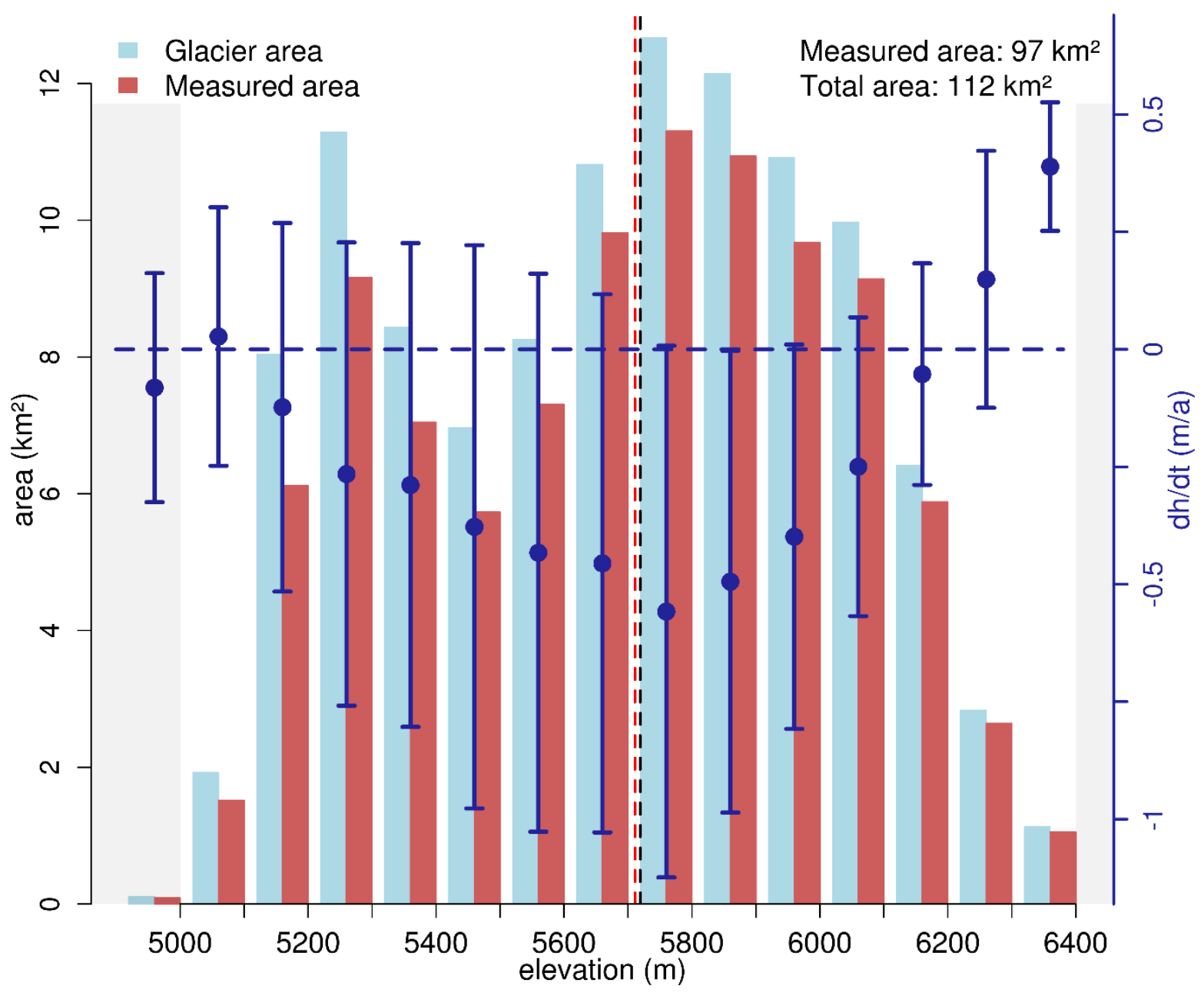

Figure S15. Hypsometric distribution of measured (red bars) and total (light blue bars) glacier area with elevation in subregion R3 in the interval 2000-2016. Blue dots represent the mean $\Delta \mathrm{h} / \Delta \mathrm{t}$ value in each elevation interval. Error bars indicate NMAD of $\Delta \mathrm{h} / \Delta \mathrm{t}$ for each hypsometric bin. Grey areas mark the lower and upper $1 \%$ quantile of the glacier area distribution. Black dashed line: mean glacier elevation; Red dashed line: equilibrium line altitude (ELA), see also Table S3. Area measurements are based on the glacier outlines from 2000, considering only regions with slopes below applied slope threshold (50, see Section 4.2) 


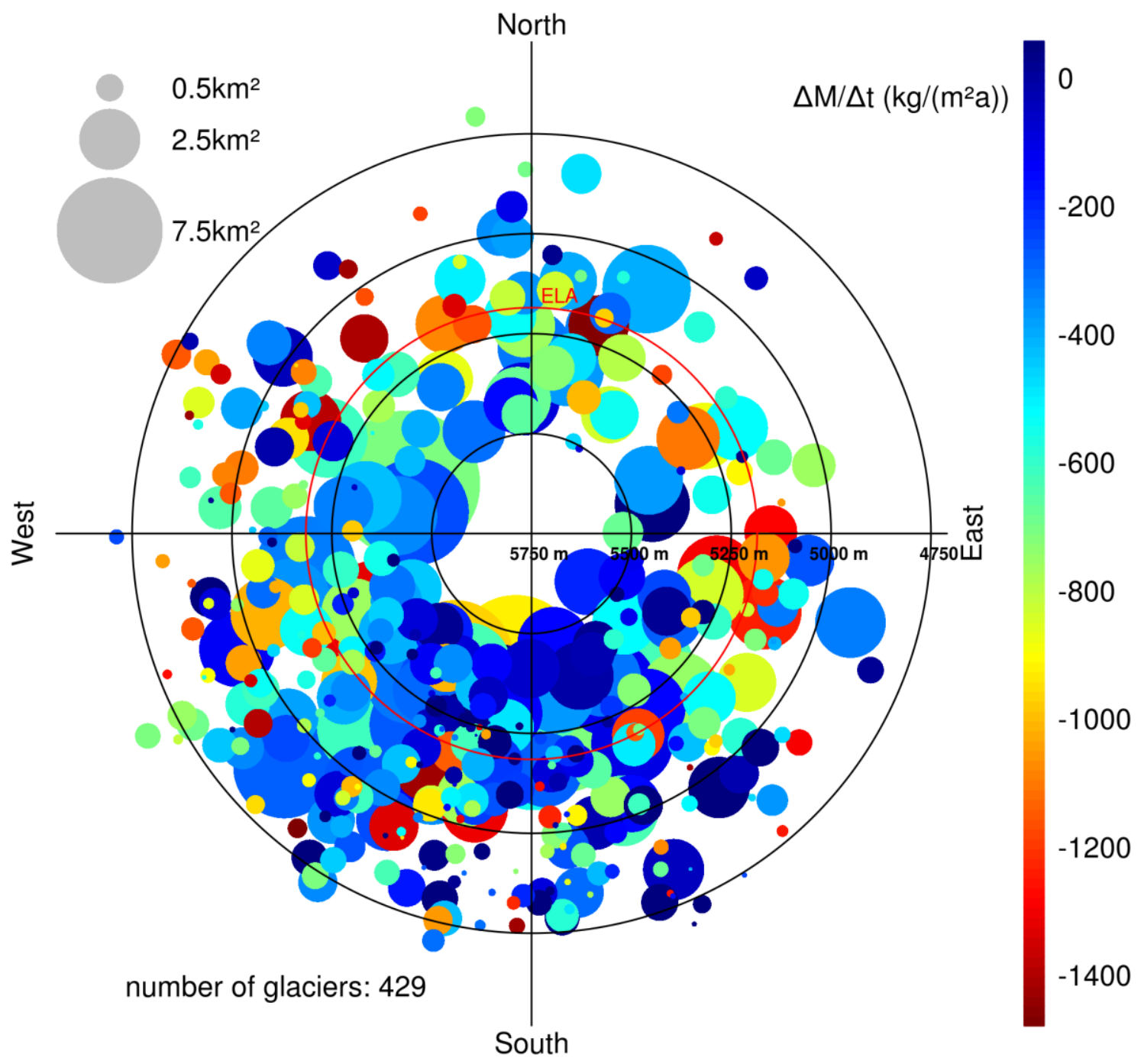

Figure S16. Polar plot of specific mass balance (dot colour) of individual glaciers in subregion R2 in the period 2000-2016 of individual glaciers. Dot size: glacier size in 2000; Radius: median elevation; Orientation: mean aspect. Red circle: equilibrium line altitude (ELA), see also Table S3. Note: only glaciers with elevation change information $>50 \%$ are included. 


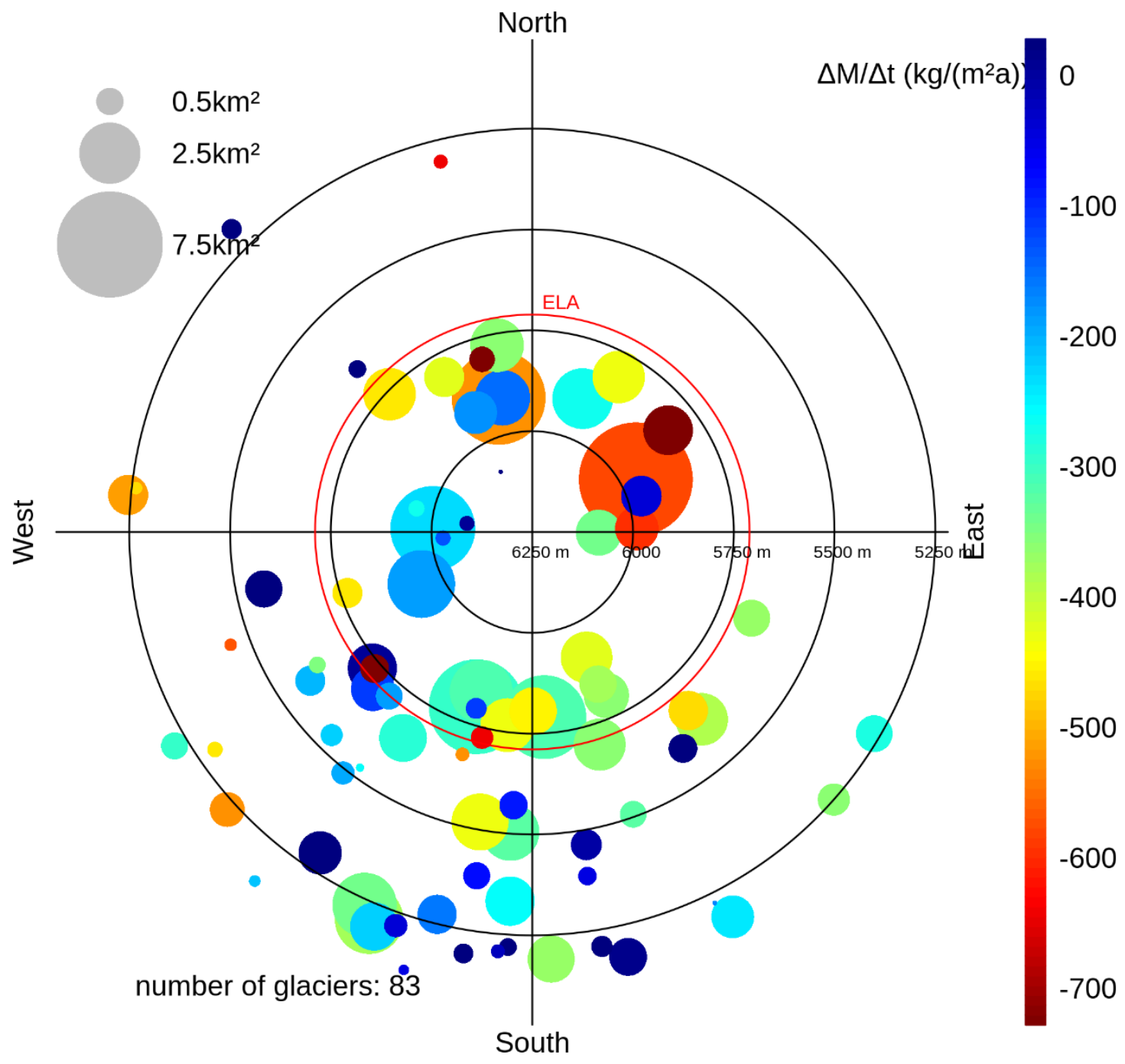

Figure S17. Polar plot of specific mass balance (dot colour) of individual glaciers in subregion R3 in the period 2000-2016 of individual glaciers. Dot size: glacier size in 2000; Radius: median elevation; Orientation: mean aspect. Red circle: equilibrium line altitude (ELA), see also Table S3. Note: only glaciers with elevation change information $>50 \%$ are included. 


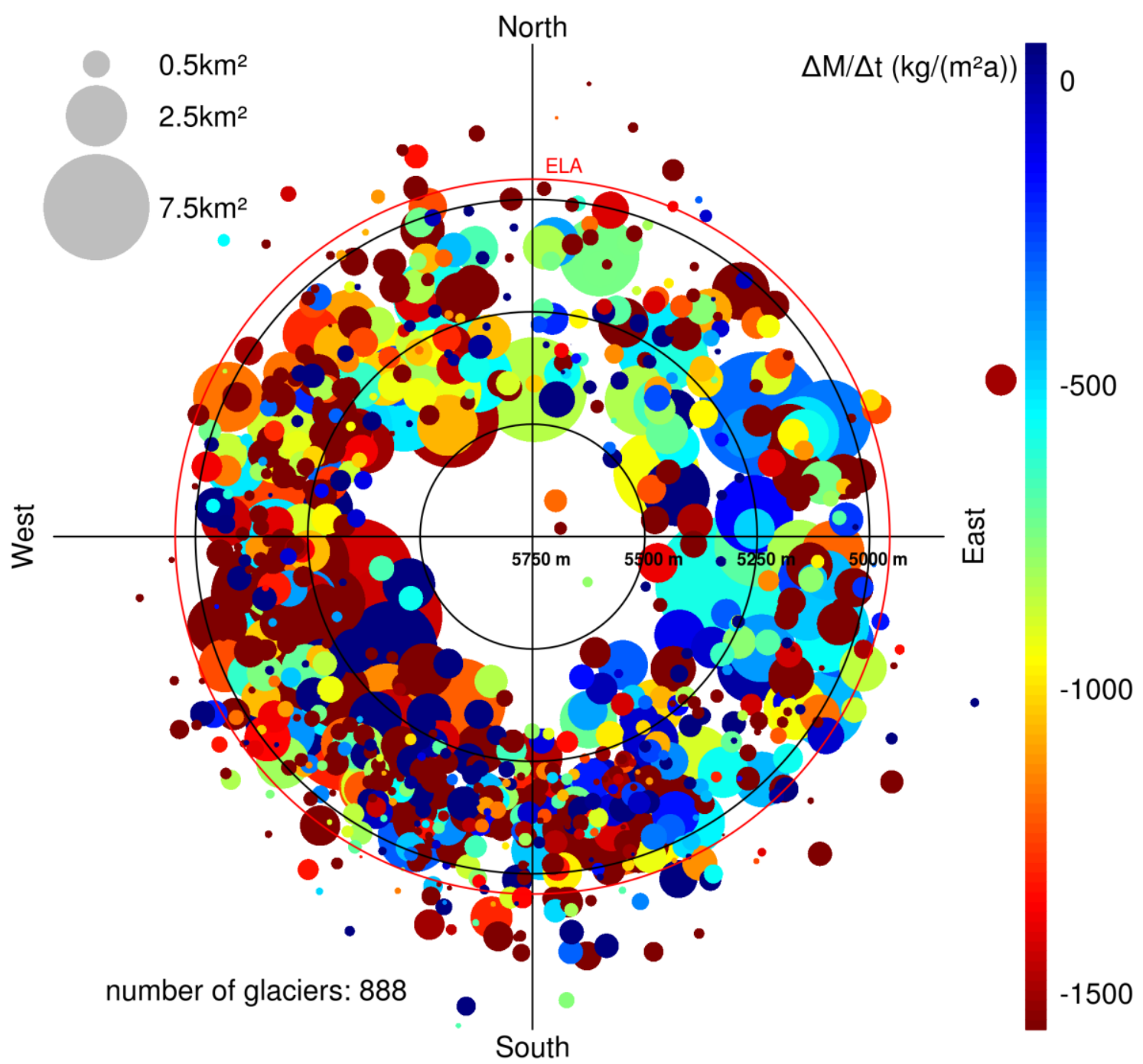

Figure S18. Polar plot of specific mass balance (dot colour) of individual glaciers in subregion R1 in the period 2013-2016 of individual glaciers. Dot size: glacier size in 2013; Radius: median elevation; Orientation: mean aspect. Red circle: equilibrium line altitude (ELA), see also Table S3. Note: only glaciers with elevation change information $>50 \%$ are included. 


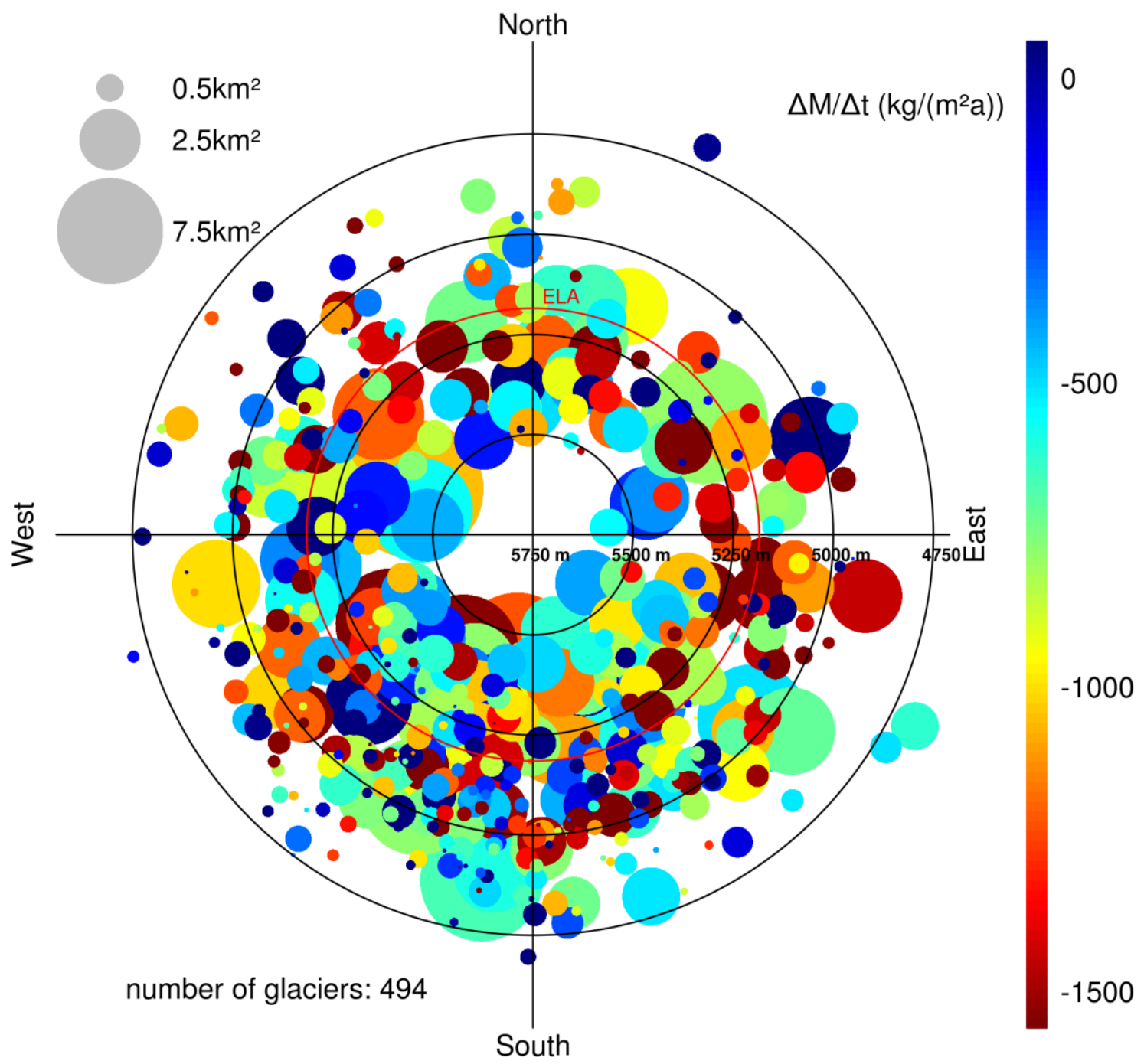

Figure S19. Polar plot of specific mass balance (dot colour) of individual glaciers in subregion R2 in the period 2013-2016 of individual glaciers. Dot size: glacier size in 2013; Radius: median elevation; Orientation: mean aspect. Red circle: equilibrium line altitude (ELA), see also Table S3. Note: only glaciers with elevation change information $>50 \%$ are included. 


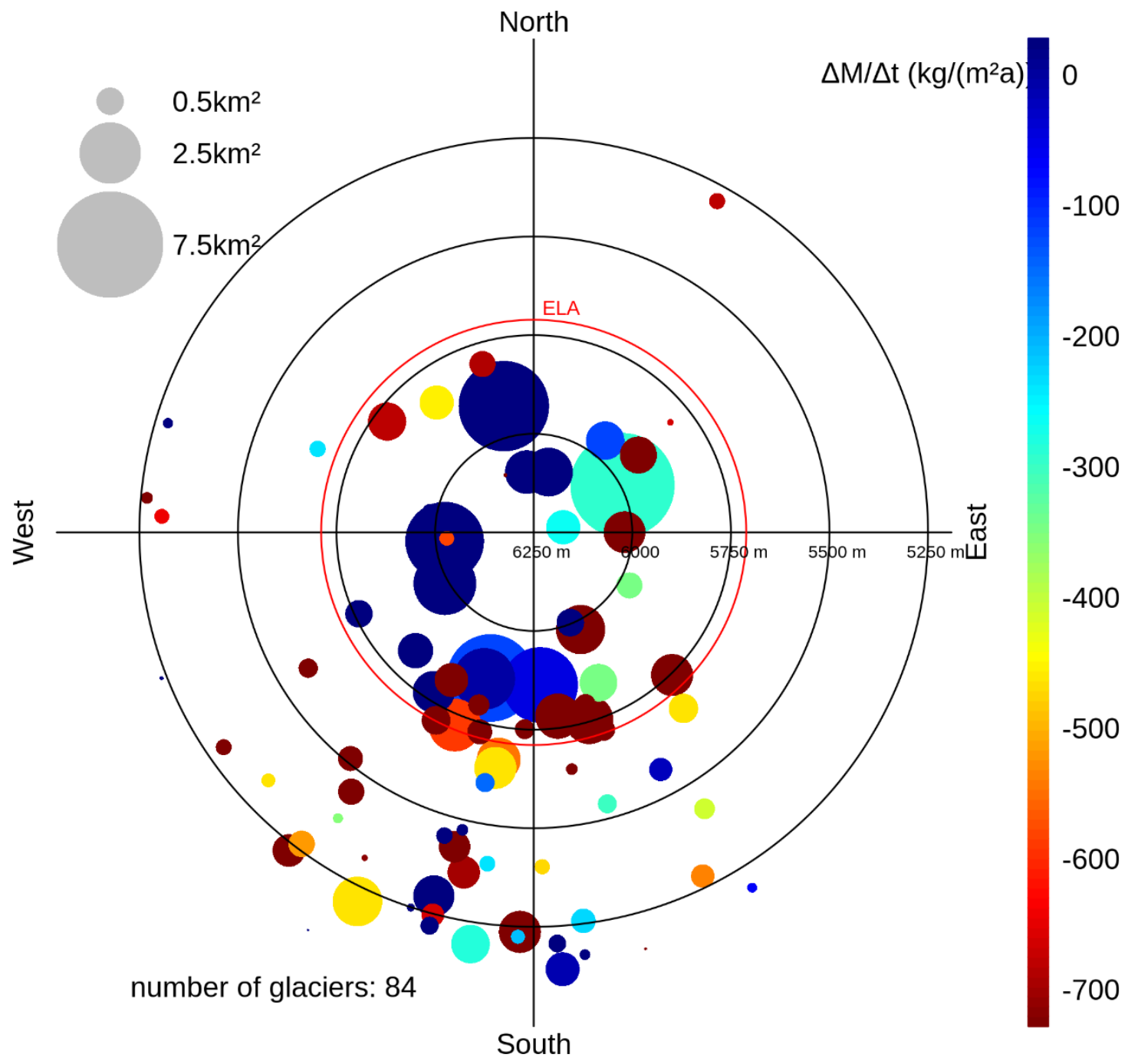

Figure S20. Polar plot of specific mass balance (dot colour) of individual glaciers in subregion R3 in the period 2013-2016 of individual glaciers. Dot size: glacier size in 2013; Radius: median elevation; Orientation: mean aspect. Red circle: equilibrium line altitude (ELA), see also Table S3. Note: only glaciers with elevation change information $>50 \%$ are included. 


\section{R1}

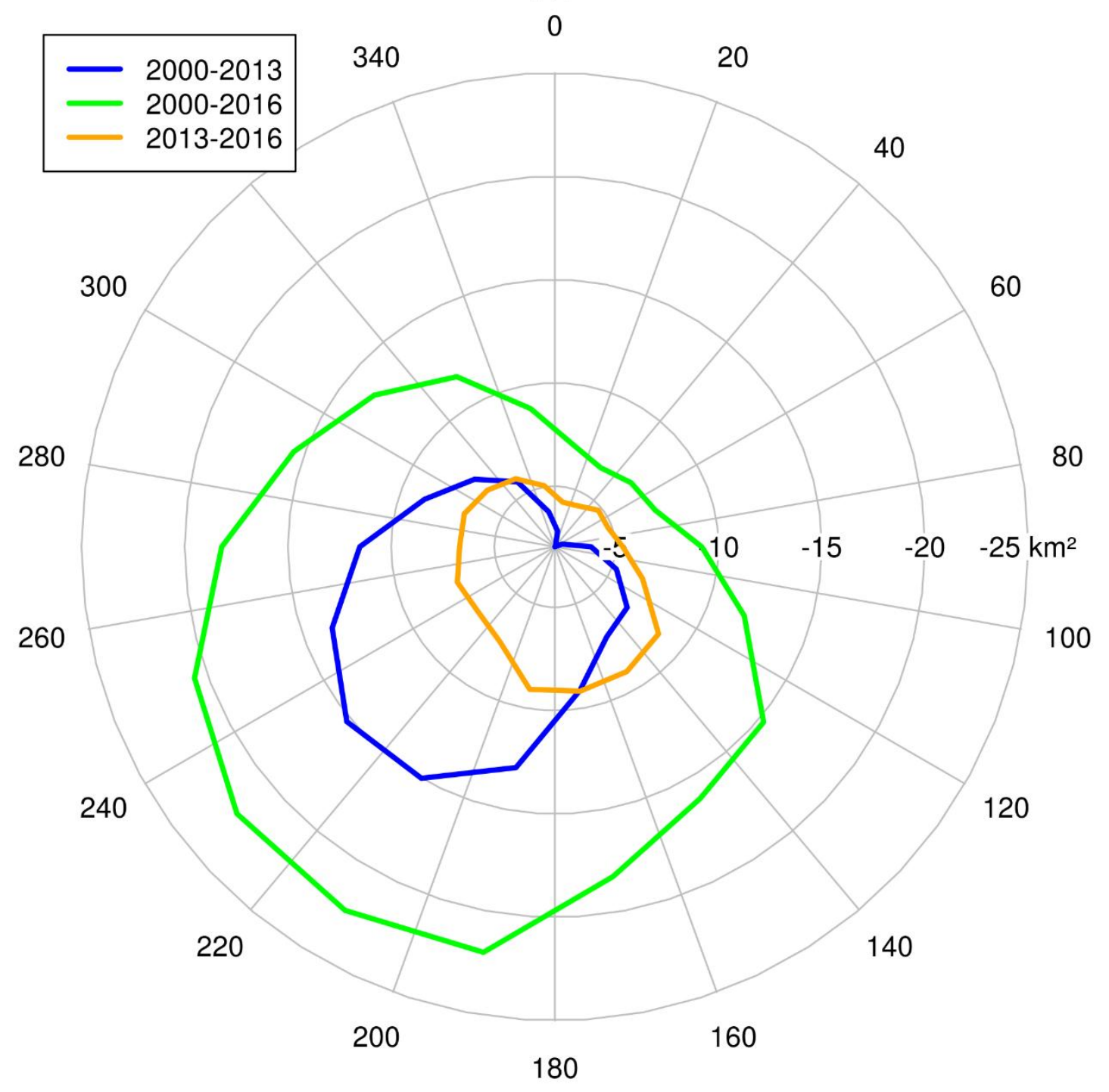

Figure S21. Polar plot of glacier area loss in subregion R1. The area losses are binned in aspect intervals of $20^{\circ}$. 


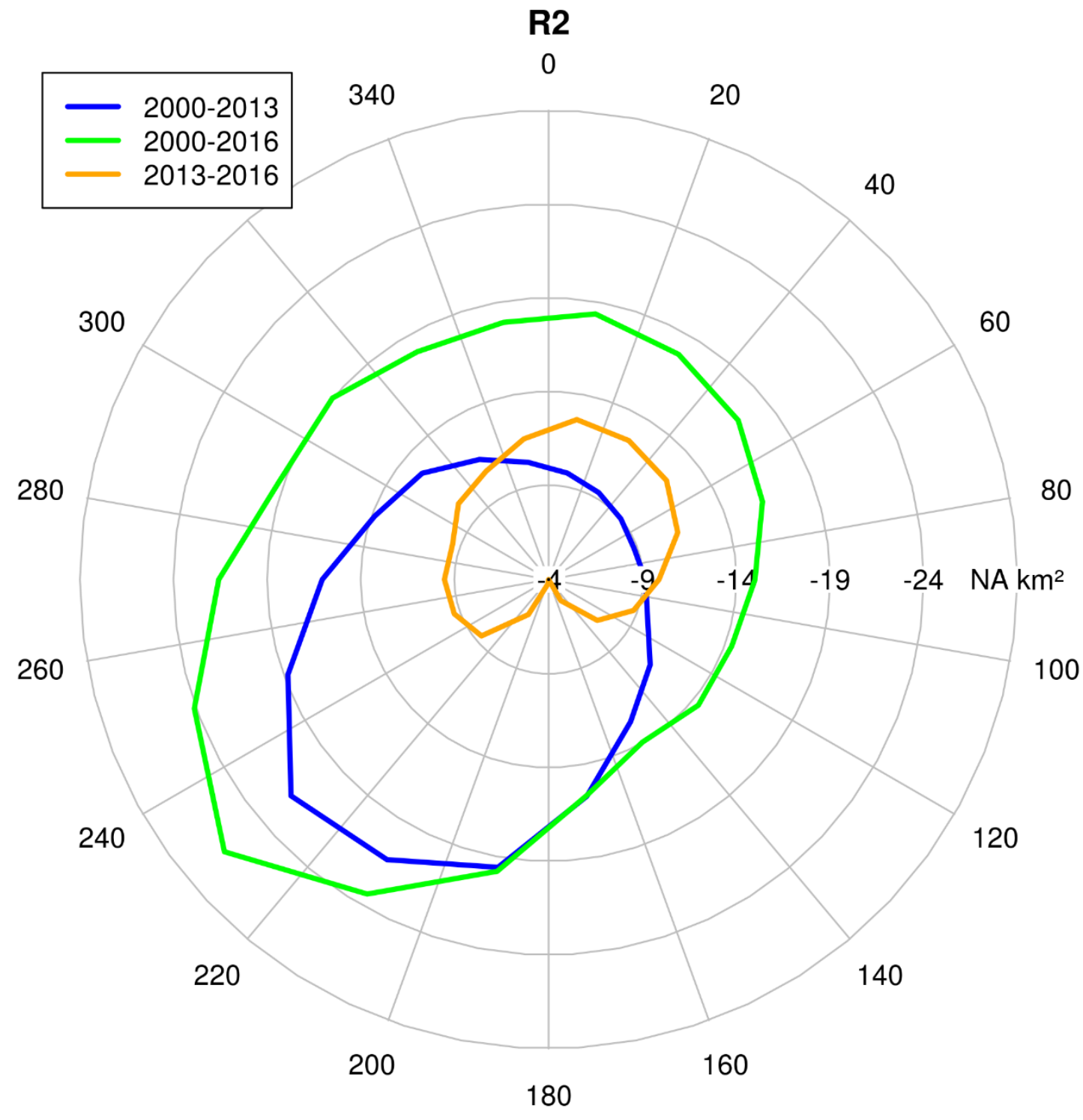

Figure S22. Polar plot of glacier area loss in subregion R2. The area losses are binned in aspect intervals of $20^{\circ}$. 


\section{R3}

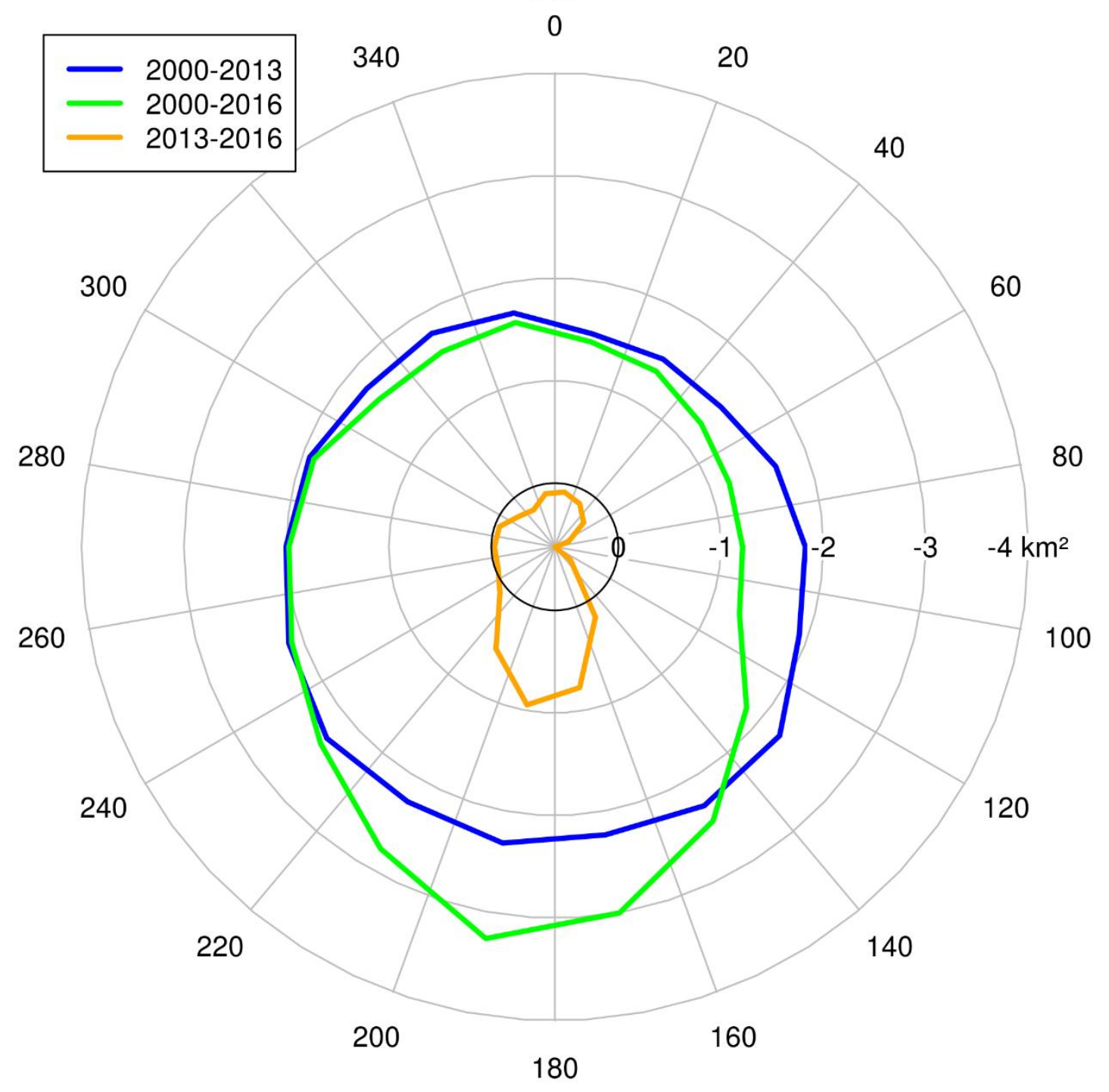

Figure S23. Polar plot of glacier area loss in subregion R3. The area losses are binned in aspect intervals of $20^{\circ}$. 

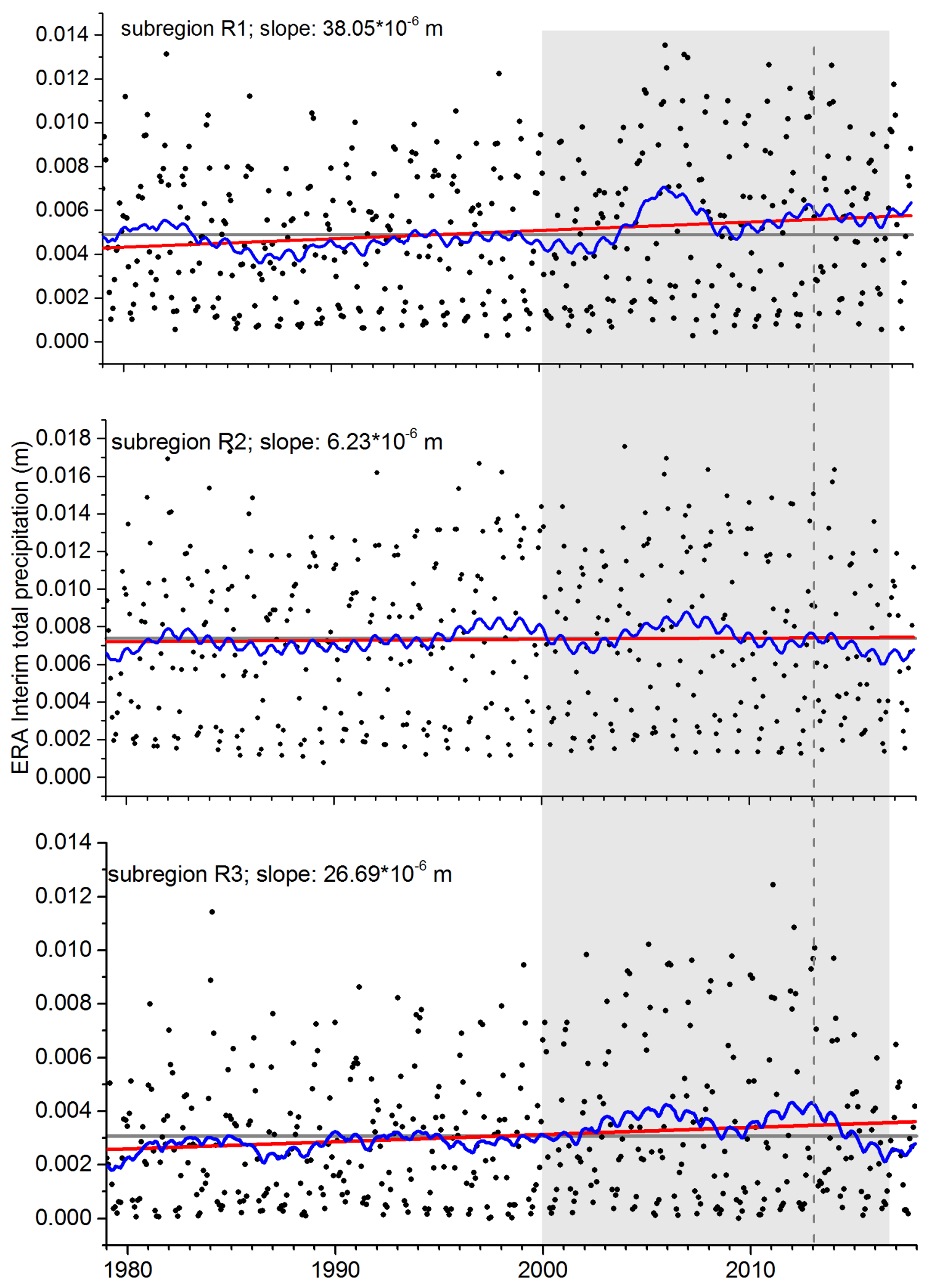

Figure S24. Total precipitation (monthly means of daily forecast accumulations) in the period 1979-2017 derived from ERA-Interim reanalysis data. Black dots: Spatial average values of glacier covered ERA-Interim grid cells in each subregion. Red line: long term trend (1979-2017), grey line: long term mean value; grey shaded area: period of mass budget and area change analysis, dashed grey line: marker for intermediate time step (early 2013) 


\section{R3-2013-2016}

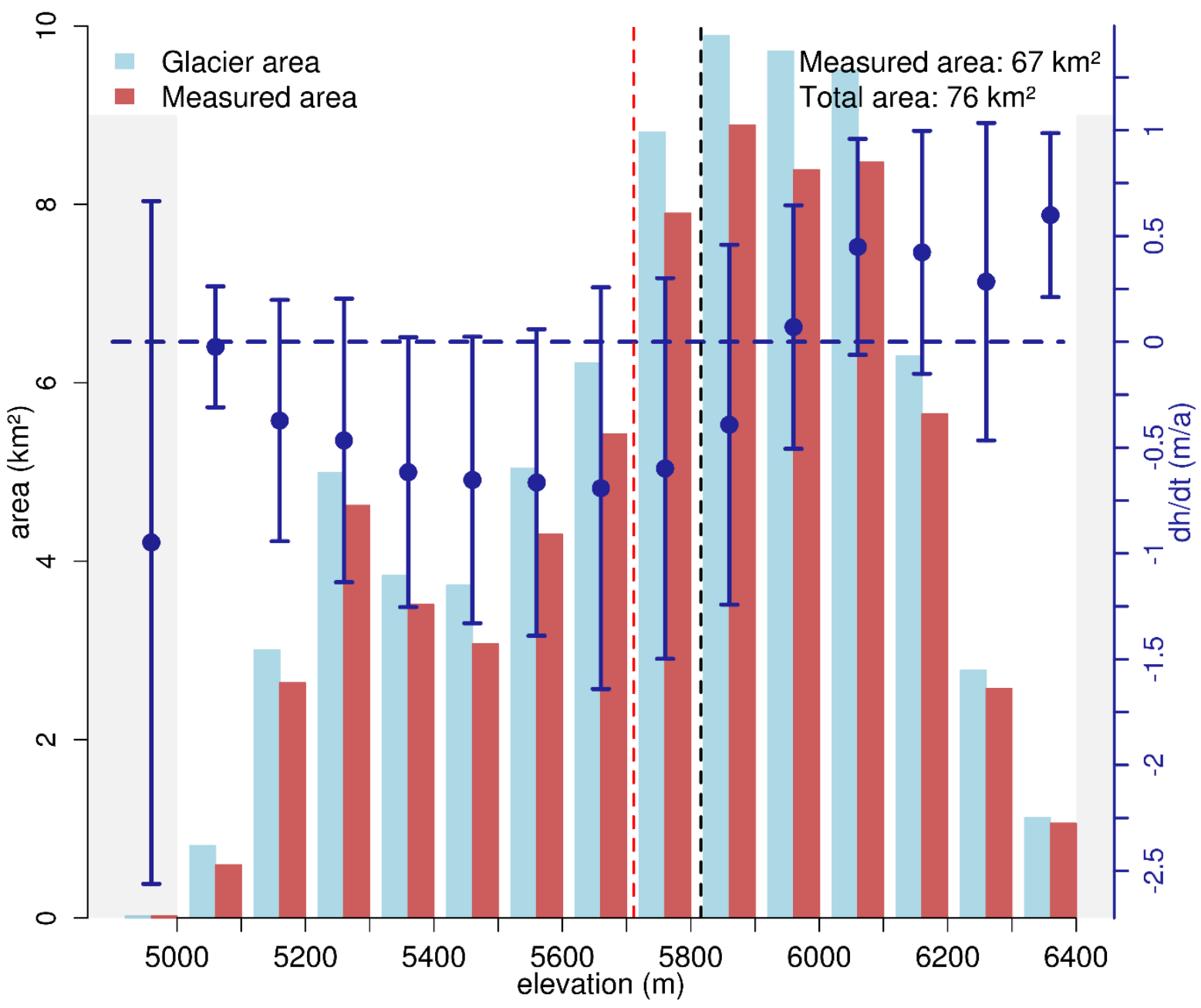

Figure S25. Hypsometric distribution of measured (red bars) and total (light blue bars) glacier area with elevation in subregion R3 in the interval 2013-2016. Blue dots represent the mean $\Delta \mathrm{h} / \Delta \mathrm{t}$ value in each elevation interval. Error bars indicate NMAD of $\Delta \mathrm{h} / \Delta \mathrm{t}$ for each hypsometric bin. Grey areas mark the lower and upper 1\% quantile of the glacier area distribution. Black dashed line: mean glacier elevation; Red dashed line: equilibrium line altitude (ELA), see also Table S3. Area measurements are based on the glacier outlines from 2013, considering only regions with slopes below applied slope threshold (50, see Section 4.2) 


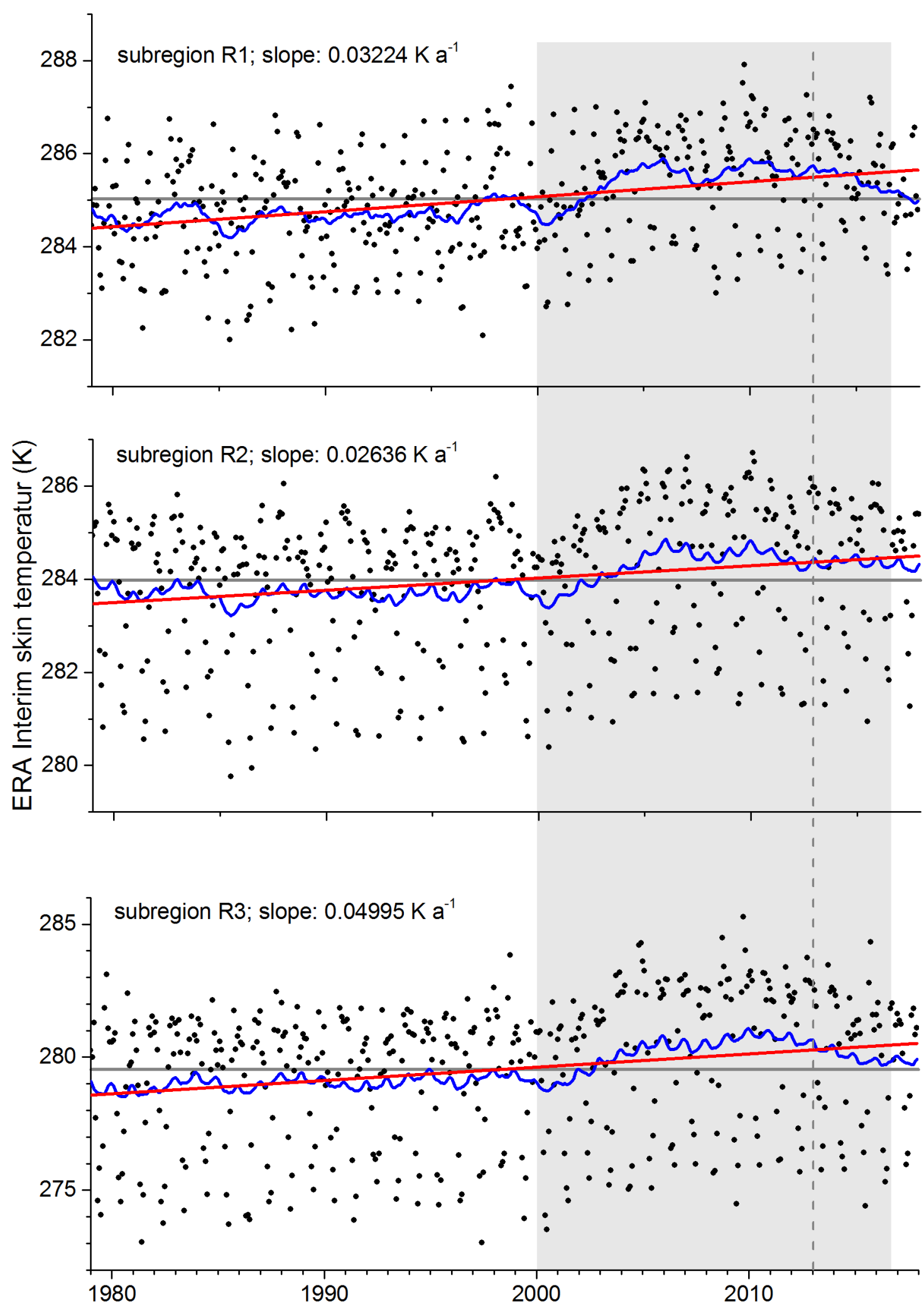

Figure S26.Skin temperature (monthly means of daily means) in the period 1979-2017 derived from ERA-Interim reanalysis data. Black dots: Spatial average values of glacier covered ERA-Interim grid cells in each subregion. Red line: long term trend (1979-2017), grey line: long term mean value; grey shaded area: period of mass budget and area change analysis, dashed grey line: marker for intermediate time step (early 2013) 


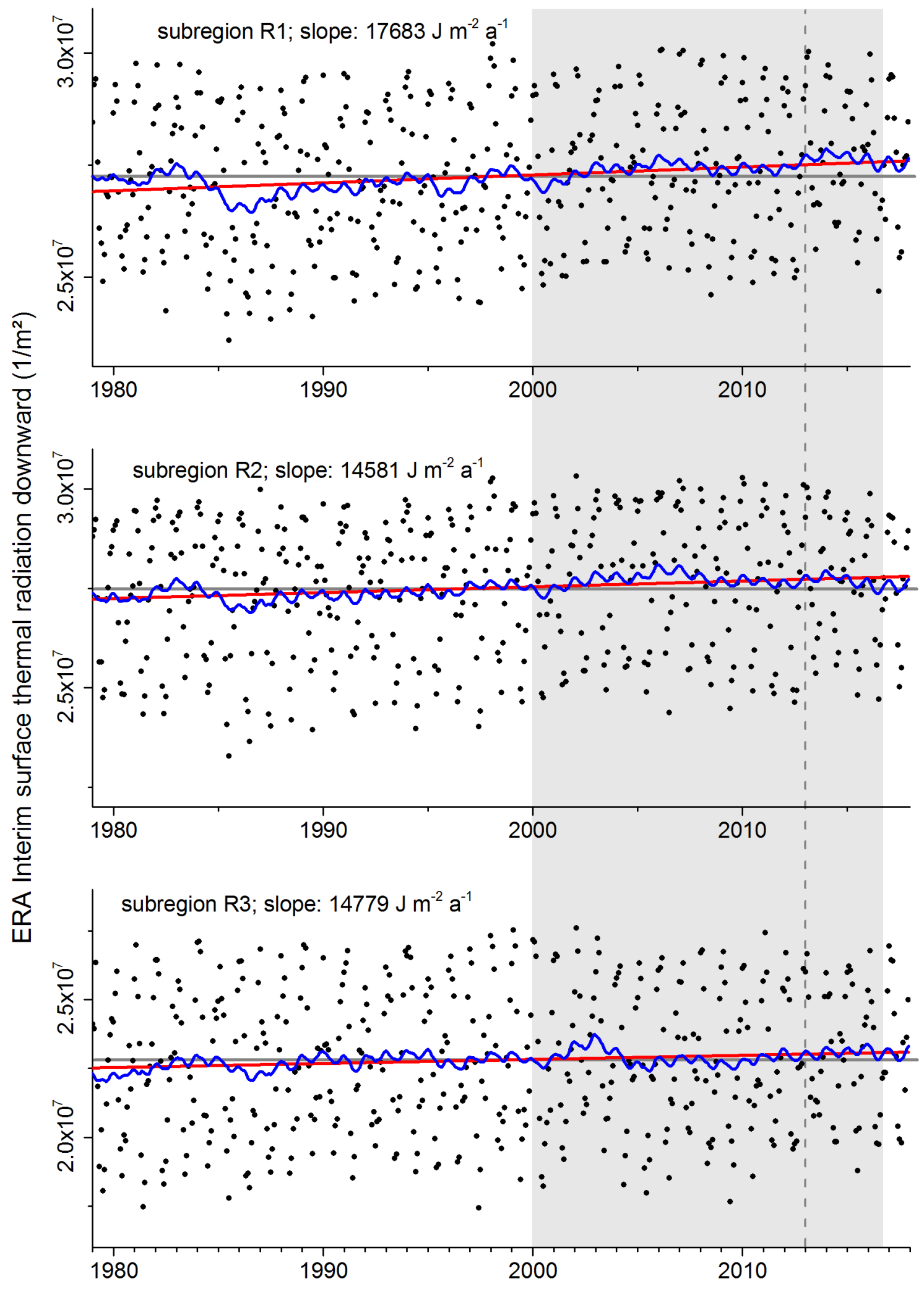

Figure S27. Surface thermal radiation downward (monthly means of daily forecast accumulations) in the period 1979-2017 derived from ERA-Interim reanalysis data. Black dots: Spatial average values of glacier covered ERA-Interim grid cells in each subregion. Red line: long term trend (1979-2017), grey line: long term mean value; grey shaded area: period of mass budget and area change analysis, dashed grey line: marker for intermediate time step (early 2013) 


\section{R1}

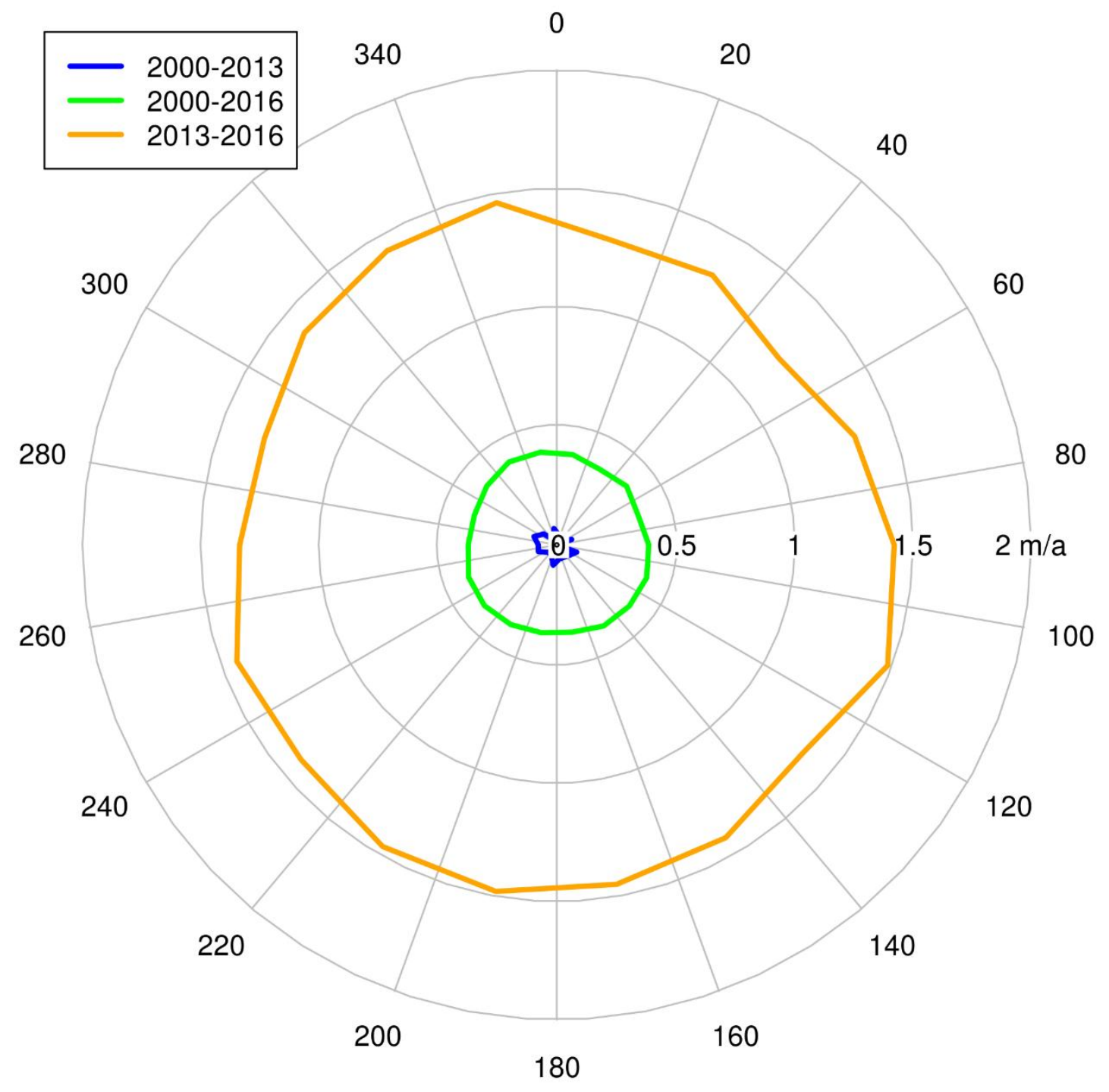

Figure S28. Polar plot of glacier surface lowering in subregion R1. The surface lowering measurements are averaged in aspect intervals of $20^{\circ}$ (mean values). 


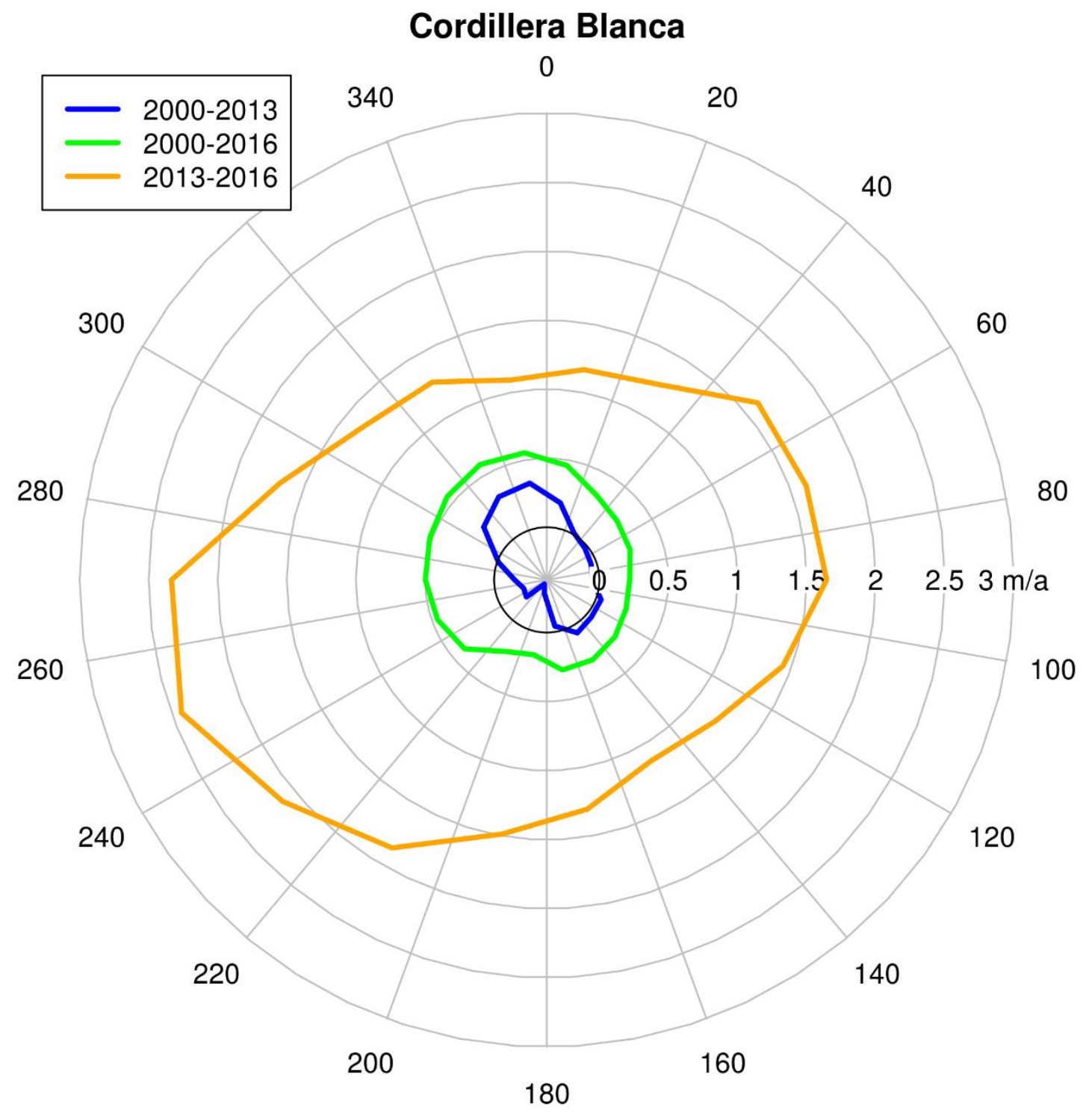

Figure S29. Polar plot of glacier surface lowering in Cordillera Blanca (subregion R1). The surface lowering measurements are averaged in aspect intervals of $20^{\circ}$ (mean values). 

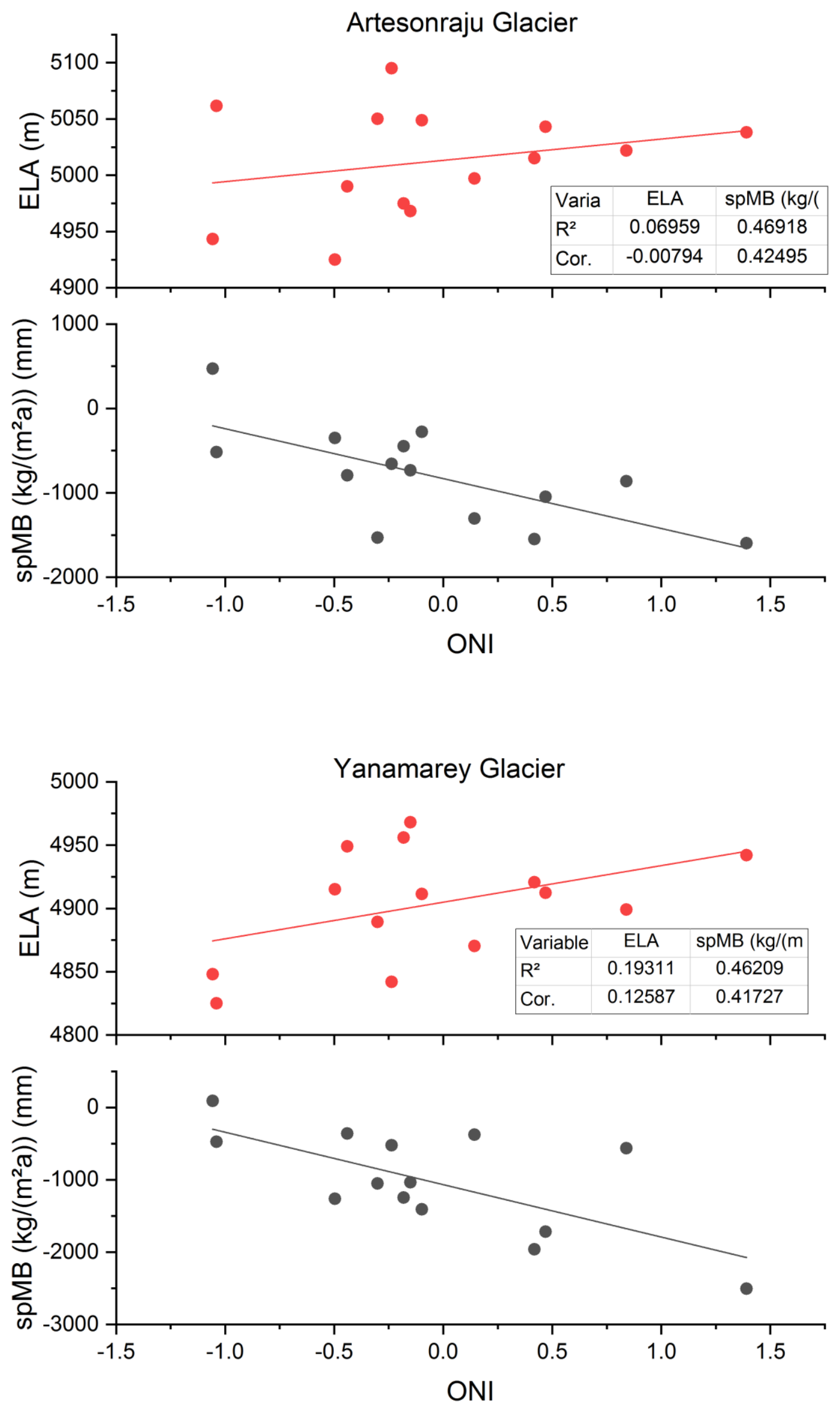

Figure S30. Annual equilibrium line altitude (ELA) and specific mass balance (spMB) derived from glaciological measurements plotted against average Oceanic Niño Index (ONI) of the respective observation periods (September-August) for Artesonraju and Yanamarey glaciers. Solid lines: linear fit of the respective variables. 


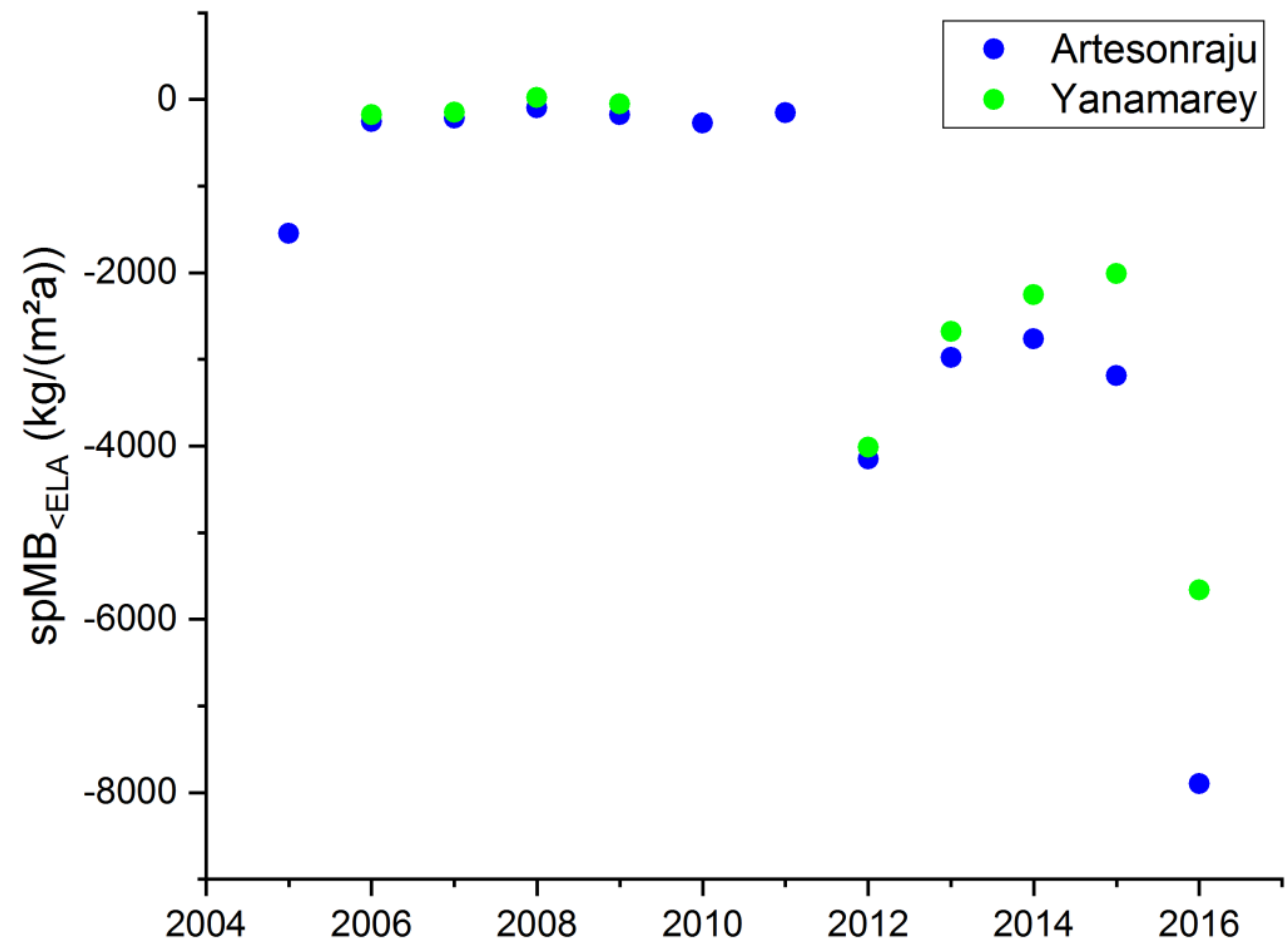

Figure S 31. Annual glaciological mass balance at regions below the ELA ( $\mathrm{spMB}<\mathrm{ELA}$ ) of Artesonraju and Yanamarey glaciers. 
Table S1. Overview of analysed TanDEM-X imagery for elevation change analysis (continued on next pages)
date
path
strip
path direction*
images

Subregion: R1 northern wet outer tropics

\begin{tabular}{|c|c|c|c|c|}
\hline 2011-12-04 & 081 & 70 & $\mathrm{D}$ & 1 \\
\hline $2011-12-25$ & 066 & 60 & $\mathrm{D}$ & 1 \\
\hline $2012-01-05$ & 066 & 50 & $\mathrm{D}$ & 3 \\
\hline 2012-01-06 & 081 & 80 & $\mathrm{D}$ & 2 \\
\hline 2012-01-11 & 157 & 70 & $\mathrm{D}$ & 1 \\
\hline 2012-01-16 & 066 & 20 & $\mathrm{D}$ & 2 \\
\hline $2012-01-22$ & 157 & 10 & $\mathrm{D}$ & 1 \\
\hline $2012-01-22$ & 157 & 10 & $\mathrm{D}$ & 3 \\
\hline $2012-01-22$ & 157 & 10 & $\mathrm{D}$ & 1 \\
\hline 2012-01-27 & 066 & 30 & $\mathrm{D}$ & 3 \\
\hline 2012-02-02 & 157 & 60 & $\mathrm{D}$ & 4 \\
\hline 2012-02-07 & 066 & 40 & $\mathrm{D}$ & 2 \\
\hline $2012-02-13$ & 157 & 80 & $\mathrm{D}$ & 2 \\
\hline 2012-02-18 & 066 & 10 & $\mathrm{D}$ & 2 \\
\hline $2012-02-24$ & 157 & 50 & $\mathrm{D}$ & 4 \\
\hline 2012-03-01 & 081 & 90 & $\mathrm{D}$ & 1 \\
\hline 2012-03-06 & 157 & 90 & $\mathrm{D}$ & 1 \\
\hline 2012-03-06 & 157 & 20 & $\mathrm{D}$ & 1 \\
\hline 2012-03-12 & 081 & 80 & $\mathrm{D}$ & 1 \\
\hline 2012-03-17 & 157 & 30 & $\mathrm{D}$ & 3 \\
\hline $2012-03-22$ & 066 & 60 & $\mathrm{D}$ & 2 \\
\hline $2012-03-23$ & 081 & 60 & $\mathrm{D}$ & 1 \\
\hline $2012-03-28$ & 157 & 40 & $\mathrm{D}$ & 2 \\
\hline $2012-03-28$ & 157 & 40 & $\mathrm{D}$ & 2 \\
\hline $2012-12-30$ & 028 & 85 & A & 4 \\
\hline 2013-01-10 & 028 & 75 & A & 6 \\
\hline 2013-01-14 & 081 & 85 & $\mathrm{D}$ & 6 \\
\hline 2013-01-21 & 028 & 65 & A & 4 \\
\hline 2013-01-24 & 066 & 45 & $\mathrm{D}$ & 2 \\
\hline 2013-01-27 & 119 & 55 & A & 1 \\
\hline 2013-01-30 & 157 & 65 & $\mathrm{D}$ & 1 \\
\hline 2013-02-01 & 028 & 55 & A & 5 \\
\hline 2013-02-04 & 066 & 55 & $\mathrm{D}$ & 2 \\
\hline 2013-02-05 & 081 & 65 & $\mathrm{D}$ & 1 \\
\hline 2013-02-10 & 157 & 45 & $\mathrm{D}$ & 2 \\
\hline 2013-02-12 & 028 & 45 & A & 1 \\
\hline 2013-02-15 & 066 & 25 & $\mathrm{D}$ & 2 \\
\hline 2013-02-21 & 157 & 35 & $\mathrm{D}$ & 2 \\
\hline $2013-02-26$ & 066 & 15 & $\mathrm{D}$ & 1 \\
\hline 2013-03-01 & 119 & 45 & A & 1 \\
\hline 2013-03-04 & 157 & 25 & $\mathrm{D}$ & 1 \\
\hline 2013-03-12 & 119 & 35 & A & 2 \\
\hline 2013-03-15 & 157 & 15 & $\mathrm{D}$ & 5 \\
\hline 2013-03-23 & 119 & 25 & A & 7 \\
\hline $2013-03-26$ & 157 & 05 & $\mathrm{D}$ & 6 \\
\hline 2016-09-08 & 119 & 10 & A & 4 \\
\hline $2016-09-13$ & 028 & 50 & $\mathrm{~A}$ & 2 \\
\hline 2016-09-16 & 066 & 10 & $\mathrm{D}$ & 2 \\
\hline 2016-09-19 & 119 & 20 & A & 1 \\
\hline 2016-09-27 & 066 & 20 & $\mathrm{D}$ & 2 \\
\hline 2016-09-30 & 119 & 30 & A & 2 \\
\hline $2016-10-08$ & 066 & 40 & $\mathrm{D}$ & 2 \\
\hline 2016-10-11 & 119 & 40 & A & 5 \\
\hline 2016-10-14 & 157 & 40 & $\mathrm{D}$ & 1 \\
\hline $2016-10-16$ & 028 & 90 & A & 5 \\
\hline 2016-10-19 & 066 & 50 & $\mathrm{D}$ & 2 \\
\hline $2016-10-22$ & 119 & 50 & A & 1 \\
\hline 2016-10-22 & 119 & 50 & A & 2 \\
\hline $2016-10-25$ & 157 & 50 & $\mathrm{D}$ & 1 \\
\hline $2016-10-27$ & 028 & 60 & $\mathrm{~A}$ & 2 \\
\hline 2016-11-02 & 119 & 60 & A & 2 \\
\hline $2016-11-16$ & 157 & 60 & $\mathrm{D}$ & 1 \\
\hline 2016-12-08 & 157 & 80 & $\mathrm{D}$ & 2 \\
\hline 2016-12-19 & 157 & 90 & $\mathrm{D}$ & 2 \\
\hline
\end{tabular}




\begin{tabular}{|c|c|c|c|c|}
\hline $2012-12-18$ & 005 & 75 & $\mathrm{D}$ & 1 \\
\hline $2012-12-29$ & 005 & 65 & $\mathrm{D}$ & 1 \\
\hline $2012-12-31$ & 043 & 85 & A & 2 \\
\hline 2013-01-04 & 096 & 75 & $\mathrm{D}$ & 3 \\
\hline 2013-01-09 & 005 & 85 & $\mathrm{D}$ & 1 \\
\hline $2013-01-10$ & 020 & 75 & $\mathrm{D}$ & 2 \\
\hline 2013-01-11 & 043 & 75 & $\mathrm{~A}$ & 3 \\
\hline 2013-01-15 & 096 & 65 & $\mathrm{D}$ & 1 \\
\hline $2013-01-15$ & 096 & 65 & $\mathrm{D}$ & 1 \\
\hline 2013-01-17 & 134 & 85 & A & 3 \\
\hline 2013-01-20 & 005 & 45 & $\mathrm{D}$ & 1 \\
\hline $2013-01-22$ & 043 & 65 & A & 1 \\
\hline $2013-01-26$ & 096 & 85 & $\mathrm{D}$ & 2 \\
\hline $2013-01-28$ & 134 & 75 & $\mathrm{~A}$ & 2 \\
\hline 2013-01-31 & 005 & 55 & $\mathrm{D}$ & 1 \\
\hline 2013-02-01 & 020 & 85 & $\mathrm{D}$ & 2 \\
\hline 2013-02-02 & 043 & 55 & A & 1 \\
\hline $2013-02-06$ & 096 & 45 & $\mathrm{D}$ & 1 \\
\hline 2013-02-08 & 134 & 65 & A & 1 \\
\hline 2013-02-13 & 043 & 45 & A & 1 \\
\hline 2013-02-17 & 096 & 35 & $\mathrm{D}$ & 1 \\
\hline 2013-02-19 & 134 & 55 & A & 2 \\
\hline $2013-02-22$ & 005 & 15 & $\mathrm{D}$ & 2 \\
\hline 2013-02-24 & 043 & 35 & A & 2 \\
\hline $2013-02-28$ & 096 & 25 & $\mathrm{D}$ & 2 \\
\hline $2013-03-02$ & 134 & 45 & A & 1 \\
\hline 2013-03-07 & 043 & 25 & A & 2 \\
\hline 2013-03-11 & 096 & 15 & $\mathrm{D}$ & 2 \\
\hline 2013-03-13 & 134 & 35 & A & 2 \\
\hline $2013-03-16$ & 005 & 05 & $\mathrm{D}$ & 2 \\
\hline $2013-03-22$ & 096 & 05 & $\mathrm{D}$ & 3 \\
\hline 2013-03-24 & 134 & 25 & A & 3 \\
\hline $2013-03-27$ & 005 & 40 & $\mathrm{D}$ & 1 \\
\hline 2013-04-02 & 096 & 70 & $\mathrm{D}$ & 1 \\
\hline 2013-04-07 & 005 & 75 & $\mathrm{D}$ & 1 \\
\hline 2013-04-18 & 005 & 10 & $\mathrm{D}$ & 2 \\
\hline 2016-09-07 & 096 & 80 & $\mathrm{D}$ & 2 \\
\hline 2016-09-09 & 134 & 20 & A & 2 \\
\hline 2016-09-12 & 005 & 10 & $\mathrm{D}$ & 2 \\
\hline 2016-09-13 & 020 & 90 & $\mathrm{D}$ & 1 \\
\hline 2016-09-14 & 043 & 30 & A & 1 \\
\hline 2016-09-18 & 096 & 10 & $\mathrm{D}$ & 1 \\
\hline 2016-09-20 & 134 & 10 & A & 3 \\
\hline 2016-09-29 & 096 & 20 & $\mathrm{D}$ & 1 \\
\hline 2016-10-01 & 134 & 30 & A & 2 \\
\hline 2016-10-04 & 005 & 40 & $\mathrm{D}$ & 1 \\
\hline 2016-10-12 & 134 & 40 & A & 2 \\
\hline $2016-10-15$ & 005 & 50 & $\mathrm{D}$ & 1 \\
\hline 2016-10-21 & 096 & 30 & $\mathrm{D}$ & 2 \\
\hline $2016-10-23$ & 134 & 50 & A & 3 \\
\hline 2016-11-18 & 020 & 80 & $\mathrm{D}$ & 1 \\
\hline $2016-11-28$ & 005 & 60 & $\mathrm{D}$ & 1 \\
\hline $2016-12-20$ & 005 & 70 & $\mathrm{D}$ & 2 \\
\hline 2016-12-26 & 096 & 70 & $\mathrm{D}$ & 2 \\
\hline \multicolumn{5}{|c|}{ Subregion: R3 dry outer tropics } \\
\hline $2012-12-02$ & 096 & 15 & $\mathrm{D}$ & 1 \\
\hline $2012-12-07$ & 005 & 10 & $\mathrm{D}$ & 1 \\
\hline $2012-12-29$ & 005 & 65 & $\mathrm{D}$ & 1 \\
\hline $2012-12-29$ & 005 & 65 & $\mathrm{D}$ & 1 \\
\hline $2012-12-31$ & 043 & 85 & A & 1 \\
\hline 2013-01-09 & 005 & 85 & $\mathrm{D}$ & 2 \\
\hline 2013-01-11 & 043 & 75 & A & 4 \\
\hline $2013-01-16$ & 119 & 85 & A & 2 \\
\hline 2013-01-20 & 005 & 45 & $\mathrm{D}$ & 3 \\
\hline $2013-01-22$ & 043 & 65 & A & 1 \\
\hline $2013-01-22$ & 043 & 65 & A & 2 \\
\hline 2013-01-31 & 005 & 55 & $\mathrm{D}$ & 3 \\
\hline 2013-02-02 & 043 & 55 & A & 2 \\
\hline
\end{tabular}




$\begin{array}{lllll}2013-02-07 & 119 & 75 & \text { A } & 2 \\ 2013-02-13 & 043 & 45 & \text { A } & 4 \\ 2013-02-19 & 134 & 45 & \text { A } & 1 \\ 2013-02-24 & 043 & 35 & \text { A } & 3 \\ 2013-03-02 & 134 & 35 & \text { A } & 1 \\ 2013-03-07 & 043 & 25 & \text { A } & 2 \\ 2013-03-10 & 081 & 25 & \text { D } & 2 \\ 2013-03-13 & 134 & 25 & \text { A } & 2 \\ 2013-03-18 & 043 & 15 & \text { A } & 2 \\ 2013-03-21 & 081 & 15 & \text { D } & 2 \\ 2013-03-24 & 134 & 15 & \text { A } & 2 \\ 2013-04-02 & 096 & 70 & \text { D } & 2 \\ 2013-04-13 & 096 & 55 & \text { D } & 2 \\ 2013-04-24 & 096 & 45 & \text { D } & 2 \\ 2014-01-04 & 134 & 05 & \text { A } & 2 \\ 2014-01-15 & 134 & 15 & \text { A } & 2 \\ 2014-01-26 & 134 & 25 & \text { A } & 2 \\ 2014-03-09 & 096 & 50 & \text { D } & 2 \\ 2014-03-20 & 096 & 60 & \text { D } & 3 \\ 2016-09-07 & 096 & 10 & \text { D } & 1 \\ 2016-09-08 & 119 & 70 & \text { A } & 2 \\ 2016-09-09 & 134 & 10 & \text { A } & 2 \\ 2016-09-12 & 005 & 20 & \text { D } & 1 \\ 2016-09-14 & 043 & 20 & \text { A } & 1 \\ 2016-09-20 & 134 & 20 & \text { A } & 4 \\ 2016-09-23 & 005 & 30 & \text { D } & 2 \\ 2016-09-29 & 096 & 20 & \text { D } & 1 \\ 2016-09-30 & 119 & 80 & \text { A } & 1 \\ 2016-10-01 & 134 & 30 & \text { A } & 2 \\ 2016-10-04 & 005 & 40 & \text { D } & 2 \\ 2016-10-11 & 119 & 90 & \text { A } & 1 \\ 2016-10-12 & 134 & 40 & \text { D } & 1 \\ 2016-10-15 & 005 & 50 & \text { D } & 3 \\ 2016-10-21 & 096 & 30 & \text { D } & 1 \\ 2016-11-28 & 005 & 80 & & 2 \\ 2016-12-26 & 096 & & & 2\end{array}$

*A - ascending, D - descending 
Table S2. Overview of analysed Landsat imagery for glacier area mapping

\begin{tabular}{|c|c|c|}
\hline Date & Path & row \\
\hline \multicolumn{3}{|c|}{ Subregion: R1 northern wet outer tropic } \\
\hline 2000-09-09 & 6 & 68 \\
\hline 2000-09-09 & 6 & 69 \\
\hline $2000-08-15$ & 7 & 67 \\
\hline $2000-08-15$ & 7 & 68 \\
\hline $2000-07-21$ & 8 & 66 \\
\hline $2000-07-21$ & 8 & 77 \\
\hline 2013-06-16 & 7 & 67 \\
\hline 2013-06-16 & 7 & 68 \\
\hline 2013-07-09 & 8 & 66 \\
\hline 2013-07-09 & 8 & 67 \\
\hline 2013-07-11 & 6 & 68 \\
\hline 2013-07-11 & 6 & 69 \\
\hline 2013-08-19 & 7 & 67 \\
\hline 2014-07-12 & 8 & 66 \\
\hline 2014-07-12 & 8 & 67 \\
\hline 2014-07-14 & 6 & 68 \\
\hline 2014-08-22 & 7 & 67 \\
\hline 2014-11-19 & 6 & 69 \\
\hline $2014-11-26$ & 7 & 68 \\
\hline 2016-01-16 & 7 & 68 \\
\hline 2016-01-16 & 7 & 69 \\
\hline $2016-06-15$ & 8 & 66 \\
\hline $2016-06-15$ & 8 & 67 \\
\hline $2016-06-17$ & 6 & 68 \\
\hline 2016-07-10 & 7 & 67 \\
\hline \multicolumn{3}{|c|}{ Subregion: R2 southern wet outer tropic } \\
\hline $2000-06-23$ & 4 & 69 \\
\hline $2000-07-18$ & 3 & 69 \\
\hline 2000-07-18 & 3 & 70 \\
\hline $2000-08-17$ & 5 & 69 \\
\hline $2000-08-28$ & 2 & 70 \\
\hline $2013-06-27$ & 4 & 69 \\
\hline 2013-06-29 & 2 & 70 \\
\hline $2013-07-22$ & 3 & 70 \\
\hline 2014-06-05 & 5 & 69 \\
\hline 2016-07-21 & 4 & 69 \\
\hline $2016-07-23$ & 2 & 70 \\
\hline 2016-07-30 & 3 & 70 \\
\hline 2016-08-29 & 5 & 69 \\
\hline \multicolumn{3}{|c|}{ Subregion: R3 dry outer tropics } \\
\hline $2000-07-25$ & 4 & 70 \\
\hline $2000-07-16$ & 5 & 70 \\
\hline $2000-08-03$ & 3 & 71 \\
\hline 2000-09-11 & 4 & 71 \\
\hline $2000-09-13$ & 2 & 71 \\
\hline 2013-09-06 & 5 & 70 \\
\hline 2013-09-08 & 3 & 71 \\
\hline 2013-09-01 & 2 & 71 \\
\hline 2013-09-15 & 4 & 70 \\
\hline $2013-10-17$ & 4 & 71 \\
\hline 2016-05-11 & 3 & 71 \\
\hline $2016-05-20$ & 2 & 71 \\
\hline $2016-07-28$ & 5 & 70 \\
\hline 2016-07-21 & 4 & 70 \\
\hline 2016-07-21 & 4 & 71 \\
\hline
\end{tabular}


Table S3: Snow line altitude (SLA) and equilibrium line altitude (ELA) reported for the study region and period. (RS: remote sensing,

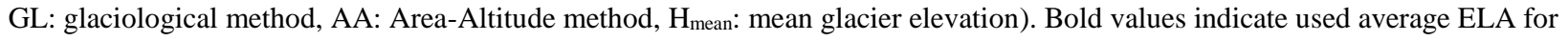
penetration depth bias estimation.

\begin{tabular}{|c|c|c|c|c|c|}
\hline period & $\begin{array}{c}\min \\
\text { (m a.s.1.) }\end{array}$ & $\begin{array}{c}\max \\
\text { (m a.s.1.) }\end{array}$ & Type & Method & Source \\
\hline \multicolumn{6}{|c|}{ Subregion R1 } \\
\hline $2000-2015$ & 4845 & 5085 & SLA & $\mathrm{RS}$ & Veettil et al., $2017 \mathrm{a}$ \\
\hline $2000-2015$ & 4720 & 4920 & SLA & $\mathrm{RS}$ & Veettil et al., 2017b \\
\hline 1999-2005 & 5034 & 5086 & SLA & $\mathrm{RS}$ & McFadden et al., 2011 \\
\hline 2006-2010 & 4835 & 5075 & SLA & $\mathrm{RS}$ & Lopez-Moreno et al., 2014 \\
\hline 2006-2008 & 4953 & 4985 & ELA & GL & Gurgiser et al., 2013 \\
\hline 2004-2015 & 4959 & 5071 & ELA & GL & Artesonraju Glacier, WGMS \\
\hline 2004-2015 & 4868 & 4942 & ELA & GL & Yanamarey Glacier, WGMS \\
\hline \multicolumn{6}{|c|}{ average: 4955} \\
\hline \multicolumn{6}{|c|}{ Subregion R2 } \\
\hline $2000-2015$ & 4680 & 5120 & SLA & $\mathrm{RS}$ & Veettil et al., 2017b \\
\hline 1998-2009 & 5526 & 5414 & SLA & $\mathrm{RS}$ & Hanshaw and Bookagen, 2014 \\
\hline 1998-2016 & 5050 & 5414 & ELA & $\mathrm{H}_{\text {mean }}$ & Drenkhan et al. 2018 \\
\hline \multicolumn{6}{|c|}{ average: 5199} \\
\hline \multicolumn{6}{|c|}{ Subregion R3 } \\
\hline $2000-2014$ & 5480 & 5745 & SLA & $\mathrm{RS}$ & Veettil et al., 2016 \\
\hline 2007 & \multicolumn{2}{|c|}{5910} & ELA & AA & Ubeda, 2011 \\
\hline
\end{tabular}

\section{References:}

Gurgiser, W., Marzeion, B., Nicholson, L., Ortner, M., Kaser, G., 2013. Modeling energy and mass balance of Shallap Glacier, Peru. The Cryosphere 7, 1787-1802. https://doi.org/10.5194/tc-7-1787-2013

Hanshaw, M.N., Bookhagen, B., 2014. Glacial areas, lake areas, and snow lines from 1975 to 2012: status of the Cordillera Vilcanota, including the Quelccaya Ice Cap, northern central Andes, Peru. The Cryosphere 8, 359-376. https://doi.org/10.5194/tc-8-359-2014

López-Moreno, J.I., Fontaneda, S., Bazo, J., Revuelto, J., Azorin-Molina, C., Valero-Garcés, B., Morán-Tejeda, E., Vicente-Serrano, S.M., Zubieta, R., Alejo-Cochachín, J., 2014. Recent glacier retreat and climate trends in Cordillera Huaytapallana, Peru. Glob. Planet. Change 112, 1-11. https://doi.org/10.1016/j.gloplacha.2013.10.010

McFadden, E.M., Ramage, J., Rodbell, D.T., 2011. Landsat TM and ETM+ derived snowline altitudes in the Cordillera Huayhuash and Cordillera Raura, Peru, 1986\&ndash;2005. The Cryosphere 5, 419-430. https://doi.org/10.5194/tc-5-4192011

Ubeda, J., 2011. El impacto del cambio climático en los glaciares del complejo volcánico Nevado Coropuna, (Cordillera Occidental de los Andes Centrales) (info:eu-repo/semantics/doctoralThesis). Universidad Complutense de Madrid, Servicio de Publicaciones, Madrid.

Veettil, B.K., Bremer, U.F., Souza, S.F. de, Maier, É.L.B., Simões, J.C., 2016. Variations in annual snowline and area of an ice-covered stratovolcano in the Cordillera Ampato, Peru, using remote sensing data (1986-2014). Geocarto Int. 31, 544-556. https://doi.org/10.1080/10106049.2015.1059902

Veettil, B.K., Wang, S., Bremer, U.F., de Souza, S.F., Simões, J.C., 2017a. Recent trends in annual snowline variations in the northern wet outer tropics: case studies from southern Cordillera Blanca, Peru. Theor. Appl. Climatol. 129, $213-227$. https://doi.org/10.1007/s00704-016-1775-0

Veettil, B.K., Wang, S., Simões, J.C., Ruiz Pereira, S.F., de Souza, S.F., 2017b. Regional climate forcing and topographic influence on glacier shrinkage: eastern cordilleras of Peru. Int. J. Climatol. 38, 979-995. https://doi.org/10.1002/joc.5226 